\title{
A SURVEY OF NIELSEN PERIODIC POINT THEORY (FIXED $n$ )
}

\author{
PHILIP R. HEATH \\ Department of Mathematics, Memorial University of Newfoundland \\ Newfoundland, A1C 5S7 Canada \\ E-mail: pheath@math.mun.ca
}

Introduction. The ordinary Nielsen number $N(f)$ of a self map $f: X \rightarrow X$ of a compact connected ANR $X$ is a lower bound for the minimum number $M(f)$ of fixed points within the homotopy class of $f$. Under certain mild assumptions this lower bound is known to be sharp, that is, there is a map $g$ homotopic to $f$ with exactly $N(f)$ fixed points. The Nielsen periodic point theory referred to in the title of this paper concerns the generalization of the above to the Nielsen type theory which attempts to determine, for a fixed $n$, a lower bound for the least number of periodic points within the homotopy class of $f$.

For the study of periodic points for fixed period $n$ there are two numbers to consider rather than one. Since for $m \mid n$, if $f^{m}(x)=x$, then $f^{n}(x)=x$ too, we want firstly, for fixed $n$, to consider all periodic points with period dividing $n$. Secondly we want to consider those periodic points of period exactly $n$. We denote the corresponding numbers due originally to Jiang [20], by $N \Phi_{n}(f)$ and $N P_{n}(f)$ respectively. They are homotopy invariant lower bounds for the respective number of periodic points.

There are three aspects to ordinary Nielsen theory, firstly there is the computation of $N(f)$ (which is in general very hard); secondly there is the 'Wecken' question, that is, the question of when $N(f)$ and $M(f)$ coincide; and finally there are the extensions of ordinary Nielsen theory to the various restricted classes (relative Nielsen theory, fibred Nielsen theory etc.). In the same way there are parallel considerations for the Nielsen type numbers $N \Phi_{n}(f)$ and $N P_{n}(f)$, and in this survey we outline the progress made in these areas.

Since the definitions are rather complicated we spend time motivating them both geometrically and algebraically. The reader might find it helpful as a first run at this material to aim to understand the two fundamental examples 1.15 and 1.16 in section

1991 Mathematics Subject Classification: Primary 55M20.

The paper is in final form and no version of it will be published elsewhere. 
1.3. The latter example shows clearly why $N P_{n}(f)$ (and hence $N \Phi_{n}(f)$ ) can be unrelated to $N\left(f^{m}\right)$ for any $m$, and why we need to use orbits rather than classes. An understanding of lemma 1.13 (and 1.6) is also essential in this regard. Examples 1.15 and 1.2 help us to understand why we can sometime relate $N \Phi_{n}(f)$ to $N\left(f^{m}\right)$ for the collection of $m$ with $m \mid n$, and so motivates us to look for conditions and algorithms for such considerations.

\section{Outline}

Introduction

1. Definitions, examples and properties

1.1. Geometric definitions

1.2. Algebraic definitions

1.3. Two fundamental examples

1.4. Properties and estimates of $N \Phi_{n}(f)$ and $N P_{n}(f)$

2. Computational theorems and calculations

2.1. Jiang spaces

2.2. Mainly for tori

2.3. Nil and solvmanifolds

2.3A. Equivariant fixed point theory and periodic points

2.3B. Fibre techniques

2.4. The Fadell-Husseini method and the Fox calculus

3. Restricted classes

3.1. Relative periodic point theory

3.2. Fibred periodic point theory

4. Progress in periodic Wecken theorems

5. Concluding comments, relationship of orbits to growth rate etc.

1. Definitions, examples and properties. Since the definitions of the numbers $N \Phi_{n}(f)$ and $N P_{n}(f)$ are not entirely straightforward we spend this section giving and motivating the definitions, giving examples and the elementary properties of these numbers. We indicate clearly, for example, why the ordinary Nielsen number $N\left(f^{n}\right)$ of the $n$th iterate of $f$ is not in general a lower bound that would have any chance of satisfying appropriate Wecken properties. We will see that part of the solution to the problems is to work with orbits rather than classes.

In contrast to the original definitions due to Jiang [20], we follow the philosophy from [7] (and taken up in [5] and [18]) that separates the algebraic from the topological (geometric) ingredients, and does this using a modified fundamental group approach.

1.1. Geometric definitions. Our context is a map $f: X \rightarrow X$ of a compact connected ANR $X$. For such an $f$ the fixed point set is denoted by $\Phi(f)$, that is, $\Phi(f)=\{x \in$ $X \mid f(x)=x\}$. We will assume the reader has some familiarity with the ordinary Nielsen number $N(f)$ and its properties, i.e. that $N(f) \leq M(f):=\{\#(\Phi(g)) \mid g \sim f\}$, and that $N(f)$ is a homotopy invariant, homotopy type invariant etc. Our aim in this subsection is to motivate and give the geometric definitions. We develop such geometric concepts as classes, orbits, reducibility etc. The next subsection will replace the geometric definitions by their algebraic counterparts, and relate the algebra to the geometry. 
A periodic point of a self map $f: X \rightarrow X$ is simply a fixed point of the $n$th iterate $f^{n}$ of $f$, i.e. an element of $\Phi\left(f^{n}\right)$. The periodic points of least period $n$ consists of the set

$$
P_{n}(f)=\left\{x \in \Phi\left(f^{n}\right) \mid \text { if } m<n \text { then } f^{m}(x) \neq x\right\} .
$$

If $x \in P_{n}(f)$ we write $\operatorname{per}(x)=n$. Clearly $\Phi\left(f^{n}\right)=\bigcup_{m \mid n} P_{m}(f)$.

ExAmple 1.1. Let $f: S^{1} \rightarrow S^{1}$ be defined by $f\left(e^{i \theta}\right)=e^{3 i \theta}$. Then

$$
\Phi\left(f^{n}\right)=\left\{e^{2 \pi i k /\left(3^{n}-1\right)} \mid k=0,1, \ldots, 3^{n}-2\right\}
$$

in particular \# $\left(\Phi\left(f^{n}\right)\right)=3^{n}-1$ (\# denotes cardinality).

Note that $n=1, k=1$, and $n=2, k=4$, and $n=4, k=40$ determine the same point $e^{\pi i}=-1$, similarly $n=2, k=1$ and $n=4, k=10$ determine $e^{\pi i / 4}$. As an example $P_{4}(f)=\Phi\left(f^{4}\right)-\Phi\left(f^{2}\right)$, and $\#\left(P_{4}(f)\right)=72$.

Example 1.1 counts points. As with ordinary Nielsen theory we need to use Nielsen classes. There are two approaches to this, the covering space approach, and the fundamental group approach. Since they determine the same partition of $\Phi\left(f^{n}\right)$ we need only one, and we choose the latter (see [20] for the covering space approach). Let $x, y \in \Phi\left(f^{n}\right)$; then $x \sim y$ iff there is a path $c: x \rightarrow y$ with $c \simeq f^{n}(c)$ rel end points. We say that $x$ is Nielsen equivalent to $y$, and denote the set of such Nielsen classes by $\Phi\left(f^{n}\right) / \sim$ with elements $\mathbf{F}^{n}$. When $n=1$ this is the usual Nielsen equivalence. In example 1.1 it is not hard to see that each point is in its own class. We shall often refer to elements $\mathbf{F}^{n}$ of $\Phi\left(f^{n}\right) / \sim$ as geometric (as opposed to algebraic) classes.

As with the case $n=1$, each element $\mathbf{F}^{n} \in \Phi\left(f^{n}\right) / \sim$ has an index (see for example $[1])$. We denote the set of essential classes (classes with non-zero index) by $\mathcal{E}\left(f^{n}\right)$. Note that $\mathcal{E}\left(f^{n}\right) \subseteq \Phi\left(f^{n}\right) / \sim$ and $\#\left(\mathcal{E}\left(f^{n}\right)\right)=N\left(f^{n}\right)$

For the case $n=1$ we have that $N(f) \leq M(f)$. By analogy our aim is to define numbers $N \Phi_{n}(f)$ and $N P_{n}(f)$ such that

$$
N \Phi_{n}(f) \leq M \Phi_{n}(f):=\min \#\left\{\Phi\left(g^{n}\right) \mid g \sim f\right\},
$$

and

$$
N P_{n}(f) \leq M P_{n}(f):=\min \#\left\{P_{n}(g) \mid g \sim f\right\} .
$$

Note, as we shall see in $1.1, M \Phi_{4}(f)=80$, and $M P_{4}(f)=72$.

The first attempts at calculating these lower bounds was given, on the Klein bottle $K^{2}$, in a widely circulated and influential (but unpublished) preprint by Benjamin Halpern [4]. Formulae were also given in [4] for the numbers $N\left(f^{n}\right)$ for all possible maps of $K^{2}$ (see theorem 2.16 for the formulae, and [2] or [12; example 5.5] for the calculation of these numbers). In his preprint Halpern implicitly equates $M \Phi_{n}(f)$ with $N\left(f^{n}\right)$. Of course $N\left(f^{n}\right)$ is a lower bound for $M \Phi_{n}(f)$, but it can be quite inadequate as the next example shows (see also 2.12).

EXAMPLE 1.2. Let $f: S^{1} \rightarrow S^{1}$ be the flip map, i.e. the map of degree -1 given by $f\left(e^{i \theta}\right)=e^{-i \theta}$. Let $n=2$. Then $f^{2}=i d$, where $i d$ denotes the identity map, and $L\left(f^{2}\right)=\chi\left(S^{1}\right)=0$ so $N\left(f^{2}\right)=0$. However any map homotopic to $f$ must have at least two fixed points and in fact $M \Phi_{2}(f)=2=N(f)$. 
To explain this, note that the number $N\left(f^{2}\right)$ is a lower bound for the least number of fixed points for all maps $k$ with $k \sim f^{2}$. Since $f^{2}=i d$, then $k$ could be a small irrational rotation of $S^{1}$, in which case the fixed point set of $k$ is empty. The point is that we need to consider the least number of periodic points of maps $g$ that are homotopic to $f$, as opposed to fixed points of maps $k$ that are homotopic to $f^{n}$ which $N\left(f^{n}\right)$ measures. Even if we restrict to maps $k$ such that $k$ is of the form $g^{n}$ for some $g$, we may still not get it right. In this same example $f^{2} \sim i d^{2}$ but $f \nsim i d$. The point is then that the homotopy invariance is with respect to homotopies of $f$ and not with respect to homotopies of higher iterates.

Another way to see that $N\left(f^{n}\right)$ is not always a suitable candidate for $N \Phi_{n}(f)$ (see also 1.16), is to notice that it does not take account of reducibility. That is that if $m \mid n$ and $f^{m}(x)=x$ then $f^{n}(x)=x$ also.

Definition 1.3. A periodic point $x \in \Phi\left(f^{n}\right)$ is said to be reducible to $m$ if $f^{m}(x)=x$. Similarly a periodic point class $\mathbf{F}^{n}$ in $\Phi\left(f^{n}\right) / \sim$ is said to be reducible to $m$ if $\mathbf{F}^{n}$ contains a periodic point class $\mathbf{F}^{m}$ in $\Phi\left(f^{m}\right) / \sim$.

Thus in 1.1 , the 4 -periodic point $e^{i \pi}$ is reducible to 1 , and $e^{\pi i / 4}$ is reducible to 2 . There is a function

$$
\gamma=\gamma_{m, n}: \Phi\left(f^{m}\right) / \sim \rightarrow \Phi\left(f^{n}\right) / \sim
$$

defined on classes $\mathbf{F}^{m}$ as follows: if $x \in \mathbf{F}^{m}$, then $\gamma\left(\mathbf{F}^{m}\right)=\mathbf{F}^{n}$, where $\mathbf{F}^{n}$ is the class of $x$ in $\Phi\left(f^{n}\right) / \sim$. It should be clear that if $k|m| n$ then $\gamma_{k, n}=\gamma_{m, n} \gamma_{k, m}$.

Definition 1.4. If $\mathbf{F}^{n} \in \Phi\left(f^{n}\right) / \sim$, the smallest positive integer $d=d\left(\mathbf{F}^{n}\right)$ to which $\mathbf{F}^{n}$ is reducible is called its depth. If $d\left(\mathbf{F}^{n}\right)=n$, we say $\mathbf{F}^{n}$ is irreducible.

Clearly a class is irreducible if it is not in the image of $\gamma_{m, n}$ for any $m \mid n$. In 1.2 for example

$$
\gamma_{1,2}:\left\{\mathbf{F}_{0}^{1}, \mathbf{F}_{1}^{1}\right\} \rightarrow\left\{\mathbf{F}^{2}\right\} .
$$

where $-1 \in \mathbf{F}_{0}^{1}, 1 \in \mathbf{F}_{1}^{1}$, and where $\mathbf{F}^{2}$ is the single class of the identity at level 2 . So $\mathbf{F}^{2}$ is reducible to either $\mathbf{F}_{0}^{1}$ or $\mathbf{F}_{1}^{1}$, and $d\left(\mathbf{F}^{2}\right)=1$. Note by this example that $\gamma$ need not be injective.

At this point we might easily guess that $N P_{n}(f)$ should be equal to \# $\left(I E C\left(f^{n}\right)\right)$ where $I E C\left(f^{n}\right)$ is the set of irreducible essential classes of $\mathcal{E}\left(f^{n}\right)$ (in fact this was given as a definition in Halpern's unpublished works see [4] and [5]). This guess certainly works for examples 1.1 and 1.2. In particular for 1.1, $\#\left(\operatorname{IEC}\left(f^{4}\right)\right)=72=M P_{4}(f)$, and for $1.2 N P_{2}(f)=0=M P_{2}(f), N P_{1}(f)=N(f)=2=M P_{1}(f)$. However the number \# $\left(I E C\left(f^{n}\right)\right)$, though it is clearly a lower bound for $M P_{n}(f)$, may also be very inadequate! To see why note that if $x \in X$ is a periodic point of least period $n$, then so also are all the points in the orbit

$$
\operatorname{Orb}(x):=\left\{x, f(x), f^{2}(x), \ldots, f^{n-1}(x)\right\} .
$$

In other words if there is one periodic point of least period $n$ then there are at least $n$ of them. On the other hand, because the elements of $\operatorname{Orb}(x)$ may not all belong to distinct Nielsen classes, the number \# $\left(I E C\left(f^{n}\right)\right)$ may turn out to be very much less than $n$. 
We will explain this more fully later, however for the moment let $\mathcal{R}(f)$ denote the Reidemeister number of $f$ (also defined later), and recall that $\#\left(\pi_{1}(X)\right) \geq \mathcal{R}(f) \geq N(f)$. So in particular $\#\left(I E C\left(f^{n}\right)\right) \leq \#\left(\pi_{1}(X)\right)$.

In subsection 1.3 we will give an example (1.16) of a map $f$ on $\mathbf{R} P^{3}$ (real projective 3 space), and an infinite number of integers $n>2$ for which there exists a single irreducible essential class $\mathbf{F}^{n}$. From the discussion above for each such $\mathbf{F}^{n}$, there are at least $n$ periodic points of least period $n$, but $\#\left(I E C\left(f^{n}\right)\right)<\# \pi_{1}\left(\mathbf{R} P^{3}\right)=2<n$. Thus the number \# $\left(\operatorname{IEC}\left(f^{n}\right)\right)$ may be a very inadequate count of the number of periodic points. The solution is to work with orbits, but since we work with classes rather than individual points we need to formulate the concept of orbit for classes.

Definition 1.5. Let $\mathbf{F}^{n} \in \Phi\left(f^{n}\right) / \sim$ be a periodic point class. Then the orbit $\left\langle\mathbf{F}^{n}\right\rangle$ of $\mathbf{F}^{n}$ is the set

$$
\left\langle\mathbf{F}^{n}\right\rangle=\left\{\mathbf{F}^{n}, f\left(\mathbf{F}^{n}\right), f^{2}\left(\mathbf{F}^{n}\right), \ldots, f^{\ell-1}\left(\mathbf{F}^{n}\right)\right\},
$$

where if $x \in \mathbf{F}^{n}$, then $f\left(\mathbf{F}^{n}\right)$ is the class of $f(x)$ etc., and where the length $\ell=\ell\left(\left\langle\mathbf{F}^{n}\right\rangle\right)$ of $\mathbf{F}^{n}$ is the least integer $\ell$ such that $f^{\ell}\left(\mathbf{F}^{n}\right)=\mathbf{F}^{n}$.

The length of the orbit of $\mathbf{F}^{n}$ is less than or equal to $n$, and the inequality can be strict. In fact in 1.16 we will see an example where for any positive integer $r$ and for $n=2^{r}$ there is an irreducible essential orbit $\mathbf{F}^{n}$ of length 1.

It is not hard to see that properties of 'depth', of being 'irreducible' or 'essential' are properties of orbits ([20], [15]). If $\left\langle\mathbf{F}^{n}\right\rangle$ is essential and $d\left(\left\langle\mathbf{F}^{n}\right\rangle\right)=d$, then the essentiality of $\left\langle\mathbf{F}^{n}\right\rangle$ may be detecting periodic points of any period $m$ with $d|m| n$. We are assured however that there are at least $d$ of them. When $d=n$ of course there is no confusion. Thus:

LEMma 1.6. If the orbit $\left\langle\mathbf{F}^{n}\right\rangle$ is essential then it detects at least $d\left(\left\langle\mathbf{F}^{n}\right\rangle\right)$ periodic points. If the orbit $\left\langle\mathbf{F}^{n}\right\rangle$ is irreducible and essential then it detects at least $n$ periodic points of least period $n$.

We are ready to give the geometric definition of $N P_{n}(f)$.

DeFinition 1.7 (Geometric definition of $N P_{n}(f)$ ). Let $I E G O_{n}(f)$ be the set of irreducible essential geometric orbits of $f$ at level $n$. Then

$$
N P_{n}(f)=n \cdot \#\left(I E G O_{n}\right) .
$$

For 1.1 it is straightforward (but perhaps a little tedious) to see that $N P_{2}(f)=6$, and $N P_{4}(f)=72$.

Since $\Phi\left(f^{n}\right)=\cup_{m \mid n} P_{m}(f)$, a second naïve guess for $N \Phi_{n}(f)$, is that $N \Phi_{n}(f)$ might be given by the number $\sum_{m \mid n} N P_{m}(f)$. However as the next example shows this may not reveal all orbits that are detectable.

EXAmple 1.8. Consider the map $f=g \vee 1: S^{3} \vee S^{2} \rightarrow S^{3} \vee S^{2}$, where $g: S^{3} \rightarrow S^{3}$ is a map of degree 2, $1: S^{2} \rightarrow S^{2}$ is the identity, and $\vee$ is the one point union. The Lefschetz number $L\left(f^{m}\right)$ of $f^{m}$ is $2-2^{m}$ so $L(f)=0$, and for every $m>1, L\left(f^{m}\right) \neq 0$. Now $\pi=0$, so from standard Nielsen theory $N(f)=0$, and if $m>1$, then $N\left(f^{m}\right)=1$.

Consider the case $n=6$; the simple connectivity of $X$ and the fact that there are fixed points at level 1 mean that there are unique non-empty classes $\mathbf{F}^{1}, \mathbf{F}^{2}, \mathbf{F}^{3}$ and $\mathbf{F}^{6}$ 
at levels 1,2,3 and 6. The last three are essential and $\mathbf{F}^{1}$ is inessential. Again by the simple connectivity of $X$ all of the classes $\mathbf{F}^{2}, \mathbf{F}^{3}$ and $\mathbf{F}^{6}$ reduce to the inessential $\mathbf{F}^{1}$, so $\sum_{m \mid n} N P_{m}(f)=0$. However since $d\left(\mathbf{F}^{2}\right)=d\left(\mathbf{F}^{3}\right)=d\left(\mathbf{F}^{6}\right)=1$, each of these classes is detecting 1 periodic point by lemma 1.6. Of course this may simply be a single fixed point in the inessential $\mathbf{F}^{1}$. In any case $M \Phi_{6}(f) \geq 1$, so the number $\sum_{m \mid n} N P_{m}(f)=0$ may fail to count periodic points detectable by index theory.

It should be clear that the above phenomenon complicates somewhat the definition of $N \Phi_{n}(f)$. We invite the reader to see [18] for further motivation for this number. We proceed now to a provisional definition.

Definition 1.9. A finite set of orbits $S=\left\{\left\langle\mathbf{F}_{i}^{m}\right\rangle: i \in I\right\}$ is said to be a set of $n$-representatives if every essential orbit with $m \mid n$ is reducible to some $\left\langle\mathbf{F}_{i}^{m}\right\rangle \in S$.

In the last example the two sets $S=\left\{\left\langle\mathbf{F}^{1}\right\rangle\right\}$ and $S^{\prime}=\left\{\left\langle\mathbf{F}^{2}\right\rangle,\left\langle\mathbf{F}^{3}\right\rangle\right\}$ are sets of 6-representatives.

The point of course, in considering sets of $n$-representatives, is that this gives us a way to count all periodic points detectable by the fundamental lemma 1.6. We count by taking the sum of the depths of the elements. However if we take the sum of the depths of the set $S^{\prime}$ in the example above, we see that we have counted too many periodic points since both of the elements of $S^{\prime}$ reduce to the orbit $\left\langle\mathbf{F}^{1}\right\rangle$. To make sure our count is not too big, we require, in the following provisional definition, that the number be the minimum taken over all possible sets of $n$-representatives.

DeFinition 1.10 (Provisional geometric definition).

$$
N \Phi_{n}(f)=\min \left\{\sum_{\left\langle\mathbf{F}_{i}^{m}\right\rangle \in S} d\left(\left\langle\mathbf{F}_{i}^{m}\right\rangle\right): S=\text { set of } n \text {-representatives }\right\} .
$$

In the last example we would in fact have $N \Phi_{6}(f)=d\left(\left\{\mathbf{F}^{1}\right\}\right)=1$. In 1.1 the set $I E P O_{0} \cup I E P O_{2} \cup I E P O_{4}$ is a set of 4-representatives so $N \Phi_{4}(f)=80$.

1.2. Algebraic definitions. The geometric definitions in the last subsection are not practical when it comes to computation. In this subsection we give the algebraic analogues of the definitions which are more suited to computation. In particular we discuss a partition of the fundamental group $\pi$ into Reidemeister classes and orbits etc. We also relate the algebra and the geometry. As usual in Algebraic Topology the algebra reflects the geometry, but not always exactly. We will see, for example, that we can only conclude that algebraic depth is less than or equal to geometric depth.

There are two approaches to the partition of $\pi$ mentioned above, however the two approaches determine exactly the same partition. For a self map $f$ of $X$, in the fundamental group approach one chooses a basepoint $x_{0}$, and a path $\omega$ from $x_{0}$ to $f\left(x_{0}\right)$. The covering space approach selects a lift $\tilde{f}$ of $f$ to the universal covering space $\tilde{X}$ of $X$. However $\tilde{f}$ is determined by, and itself determines, a basepoint $x_{0}$ and a path $\omega$ from $x_{0}$ to $f\left(x_{0}\right)$. With this in hand the partitions determined on the one hand by $\tilde{f}$, and by $x_{0}$ and $f\left(x_{0}\right)$ on the other are exactly the same partition of $\pi$ (e.g. [6]).

We use the modified fundamental group approach used in [15] and [18], as opposed to the covering space approach used in [20]. The modification refers to the assigning 
of an index to the Reidemeister classes ([7]) which gives exactly the same index as is defined in the covering space approach. For simplicity of exposition in this survey we make the (unnecessary) assumption that there is a fixed point $x_{0}$ of $f$, so that $f_{*}^{n}$ induces a homomomorphism on $\pi_{1}\left(X, x_{0}\right)$.

Definition 1.11. Let $f: X \rightarrow X$ and let $n \geq 1$. We say that $\alpha \sim \beta$ (Reidemeister equivalent) if there exists $\delta \in \pi_{1}\left(X, x_{0}\right)$ with $\alpha=\delta \beta f_{*}^{n}\left(\delta^{-1}\right)$.

The Reidemeister class containing $\alpha$ will be denoted by $[\alpha]^{n}$. The set of all Reidemeister classes for $f_{*}^{n}$ is denoted by $\mathcal{R}\left(f_{*}^{n}\right)$. This replaces the notation $\operatorname{Coker}\left(1-f_{*}^{n x_{0}}\right)$ used in [15] and [18] (and elsewhere). The symbol $\mathcal{R}\left(f^{n}\right)$ denotes the Reidemeister number $\# \mathcal{R}\left(f_{*}^{n}\right)$ of $f^{n}$ (no $*$ in $\mathcal{R}\left(f^{n}\right)$ ). We refer to elements of $\mathcal{R}\left(f_{*}^{n}\right)$ as algebraic (as opposed to geometric) classes.

The following is an exact sequence of sets $([7])$ :

$$
\operatorname{Fixf}_{*}^{n} \stackrel{i_{*}}{\rightarrow} \pi_{1}(X) \stackrel{1 \cdot f^{-n}}{\rightarrow} \pi_{1}(X) \rightarrow \mathcal{R}\left(f_{*}^{n}\right),
$$

where $1 \cdot f^{-n}$ is the function which takes an element $\alpha \in \pi_{1}(X)$ to $\alpha f_{*}^{n}\left(\alpha^{-1}\right), F i x f_{*}^{n}$ is the subgroup $\left\{\alpha \in \pi_{1}(X) \mid f_{*}^{n}(\alpha)=\alpha\right\}$ of $\pi_{1}(X)$, and the last arrow is the canonical projection. When $\pi_{1}(X)$ is abelian, $\mathcal{R}\left(f_{*}^{n}\right)$ has a canonical group structure (e.g. [20]). In addition the sequence above becomes an exact sequence of groups and homomorphisms which is isomorphic (using functions induced by the Hurewicz homomorphism) to the following exact sequence of groups in homology (see [7]):

$$
\operatorname{ker}\left(1-f_{*}^{n}\right) \stackrel{i_{*}}{\rightarrow} H_{1}(X) \stackrel{1-f_{*}^{n}}{\rightarrow} H_{1}(X) \rightarrow \operatorname{Coker}\left(1-f_{*}^{n}\right) .
$$

Thus in example 1.1 we see that $\mathcal{R}\left(f_{*}^{1}\right) \cong \mathbf{Z}_{2}, \mathcal{R}\left(f_{*}^{2}\right) \cong \mathbf{Z}_{8}$ and $\mathcal{R}\left(f_{*}^{4}\right) \cong \mathbf{Z}_{80}$.

The algebraic and geometric theory are related by an injective function

$$
\rho=\rho_{n}: \Phi\left(f^{n}\right) / \sim \rightarrow \mathcal{R}\left(f_{*}^{n}\right),
$$

defined on a class $\mathbf{F}^{n}$ by selecting a representative $x \in \mathbf{F}^{n}$, and a path $c: x \rightarrow x_{0}$ (the basepoint) and defining $\rho\left(\mathbf{F}^{n}\right)=\left[f^{n}\left(c^{-1}\right) c\right]^{n}$. In example 1.1, taking $x_{0}=e^{i 0}$ as basepoint and $c$ as the shortest clockwise path from $e^{2 \pi i k /\left(3^{n}-1\right)}$ to $x_{0}$, we have $\rho\left(e^{2 \pi i k /\left(3^{n}-1\right)}\right)=$ $[k]^{n}$.

We think of $\rho$ as assigning a 'coordinate' to the geometric classes. As we have mentioned, the functions $\rho$ are injective, but they are not always surjective. If a class $[\alpha]^{n}$ is not in the image of $\rho$, we will think of $[\alpha]^{n}$ as the coordinate of an empty geometric class. An index can be assigned to the Reidemeister classes in such a way that the index of the possibly empty classes is zero. This is the heart of the modifications to the usual fundamental group approach mentioned above. The index $i\left([\alpha]^{n}\right)$ is assigned to the algebraic classes $[\alpha]^{n}$ as follows.

$$
i\left([\alpha]^{n}\right)= \begin{cases}\operatorname{ind}\left(\mathbf{F}^{n}\right) & \text { if }[\alpha]^{n}=\rho\left(\mathbf{F}^{n}\right), \\ 0 & \text { otherwise. }\end{cases}
$$

As with the geometric classes, an algebraic class is essential provided it has non-zero index. This should not appear too strange since this assigning of an index to a coordinate is exactly what is done in Reidemeister trace theory. To relate this to the covering space approach we note that if $x_{0} \in \Phi(f)$, then one chooses 'coordinates' by simply specifying 
the lift $\tilde{f}^{n}$ as that lift of $f^{n}$ that takes the constant path at $x_{0}$ to itself (this requires that we think of $\tilde{X}$ as equivalence classes of paths starting at $\left.x_{0}\right)$. This automatically provides $\tilde{f}^{n}$ as the coordinate of $f^{n}$, and $i\left([\alpha]^{n}\right)=i n d\left(p\left(\Phi\left(\alpha^{-1} \circ \tilde{f}^{n}\right)\right)\right)$.

The point of the definition is, of course, that the index of $[\alpha]^{n}$ is the same as the index of the geometric class (empty or not) that determines it. Since the periodic point classes are in one to one correspondence with the coordinates we are in this way able to deal with possibly empty classes without bringing in the machinery of covering spaces ([7], [15], [18]).

With this definition the function $\rho$ is index preserving, and of course

$$
N\left(f^{n}\right) \leq \mathcal{R}\left(f^{n}\right) .
$$

Corresponding to the geometric 'boosting functions' $\gamma_{m, n}$, we have 'algebraic boosting functions' $\iota_{m, n}: \mathcal{R}\left(f_{*}^{m}\right) \rightarrow \mathcal{R}\left(f_{*}^{n}\right)$ defined by the rule

$$
\iota_{m, n}\left([\alpha]^{m}\right)=\left[f_{*}^{n-m}(\alpha) f_{*}^{n-2 m}(\alpha) \ldots f_{*}^{m}(\alpha) \alpha\right]^{n} .
$$

By analogy with the geometric case for $k|m| n$ we have $\iota_{k, n}=\iota_{m, n} \iota_{k, m}$.

An easy calculation shows that $\iota_{m, n}\left(\left[f^{m}\left(c^{-1}\right) c\right]^{m}\right)=\left[f^{n}\left(c^{-1}\right) c\right]^{n}$, that is, that the left hand diagram below is commutative for all $m \mid n$.

$$
\begin{array}{lllll}
\Phi\left(f^{m}\right) / \sim \stackrel{\rho}{\rightarrow} \mathcal{R}\left(f_{*}^{m}\right) & \Phi\left(f^{m}\right) / \sim \stackrel{\rho}{\rightarrow} \mathcal{R}\left(f_{*}^{m}\right) \\
\gamma_{m, n} \downarrow & \iota_{m, n} \downarrow & f_{*} \downarrow & & f_{*} \downarrow \\
\Phi\left(f^{n}\right) / \sim \stackrel{\rho}{\rightarrow} \mathcal{R}\left(f_{*}^{n}\right) & \Phi\left(f^{m}\right) / \sim \stackrel{\rho}{\rightarrow} \mathcal{R}\left(f_{*}^{m}\right)
\end{array}
$$

It is not hard to see that the right hand diagram is also commutative for all $m$, where $f_{*}: \mathcal{R}\left(f_{*}^{m}\right) \rightarrow \mathcal{R}\left(f_{*}^{m}\right)$ is defined by $f_{*}\left([\alpha]^{m}\right)=\left[f_{*}(\alpha)\right]^{m}$. Note that $f_{*}$ is an index preserving bijection ([15]). We are now ready to make the algebraic analogues of the geometric definitions. For example:

Definition 1.12. Let $f: X \rightarrow X$ be a map. We say $[\alpha]^{n} \in \mathcal{R}\left(f_{*}^{n}\right)$ is reducible to $[\beta]^{m} \in \mathcal{R}\left(f_{*}^{m}\right)$ if $\iota_{m, n}\left([\beta]^{m}\right)=[\alpha]^{n}$. We say that $[\alpha]^{n}$ has depth $d$ if $d$ is the smallest integer for which there is a class $[\delta]^{d}$ to which $[\alpha]^{n}$ reduces.

The point of the algebraic definitions is that many times it is only from the algebra that we are able to detect the corresponding geometric classes.

We will not give all the algebraic analogues of the geometric definitions in detail. It should be obvious for example that just as the function $f$ defines orbits on the Nielsen classes $\Phi\left(f^{n}\right) / \sim, f$ also induces orbits on the Reidemeister classes by the function $f_{*}$ defined above. From the injectivity of $\rho$, and the right hand diagram above, the geometric length of orbit and the algebraic length coincide (see [15]). On the other hand from the left hand diagram we can only deduce that algebraic depth is less than or equal to geometric depth. However from 1.6 we do have:

LEMmA 1.13. If the orbit $\left\langle[\alpha]^{n}\right\rangle$ is essential and has (algebraic) depth $d$ then this orbit detects at least $d$ periodic points.

Obviously we do not always know the geometry, and so must rely on the algebra. It is to make this formal that we now turn to 
DeFinition 1.14. We define the concepts of, reducible to $m$, sets of $n$-representatives exactly as in the geometric definitions but with the corresponding geometric ingredients replaced by their algebraic counterparts. In particular we have the fully fledged definitions of $N P_{n}(f)$ and $N \Phi_{n}(f)$ as follows:

$$
N P_{n}(f)=n \cdot \#\left(I E O_{n}\right),
$$

where $I E O_{n}(f)$ is the set of irreducible essential algebraic orbits of $f$ at level $n$. Furthermore

$$
N \Phi_{n}(f)=\min \left\{\sum_{\left\langle[\alpha]_{i}^{m}\right\rangle \in S} d\left(\left\langle[\alpha]_{i}^{m}\right\rangle\right): S=\text { algebraic set of } n \text {-representatives }\right\} .
$$

1.3. Two fundamental examples. We now illustrate the power of the algebraic definitions by giving two examples of calculations of $N P_{n}(f)$ and $N \Phi_{n}(f)$. The second example will emphasize the advantage of the algebraic approach. For the first illustration we simply rework example 1.1 in its algebraic form.

EXAMPLE 1.15. Recall from above for the standard map of $S^{1}$ of degree three (example 1.1) that $\mathcal{R}\left(f_{*}^{1}\right) \cong \mathbf{Z}_{2}, \mathcal{R}\left(f_{*}^{2}\right) \cong \mathbf{Z}_{8}$ and $\mathcal{R}\left(f_{*}^{4}\right) \cong \mathbf{Z}_{80}$. Since we are working with abelian groups we denote the binary operation by + . Note then that $\iota_{1,2}(\alpha)=f(\alpha)+\alpha=$ $3 \alpha+\alpha$, that is, $\iota_{1,2}$ is multiplication by $3+1=4$. Similarly $\iota_{2,4}$ is multiplication by $3^{2}+1$ $=10$, while $\iota_{1,4}$ is multiplication by $3^{3}+3^{2}+3^{1}+1=40$. This is illustrated in the diagram below:

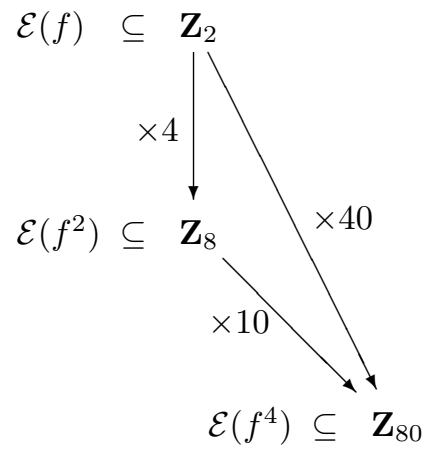

The fact that $4 \times 10=40$ illustrates the relationship between the $\iota\left(\iota_{k, n}=\iota_{m, n} \iota_{k, m}\right)$ mentioned earlier. The inclusions are of course induced by the various $\rho$. Since $S^{1}$ is a Jiang space and $L\left(f^{j}\right) \neq 0$ for all $j$, we see that the inclusions are in fact bijections, so all the algebraic classes are essential in the sense defined above. Thus we need only deal with the algebra.

Using the fact that on $\mathcal{R}\left(f_{*}^{n}\right)$ the function $f_{*}$ is multiplication by 3 , we see that at level 4 there are 18 irreducible essential orbits, at level 2 there are three, and at level 1 just 2. It is not hard to see that this set is a minimal set of 4 representatives, and $N P_{1}(f)=2, N P_{2}(f)=6$ and $N P_{4}(f)=72$. Also $N \Phi_{4}(f)=1 \cdot 2+2 \cdot 3+4 \cdot 18=80$. Note in this example that $N P_{n}(f)=\#\left(I E C_{n}(f)\right)$ (see $\left.1.19(\mathrm{v})\right)$, and $N \Phi_{n}(f)=N\left(f^{n}\right)$.

The next example formalizes the one on $\mathbf{R} P^{3}$ hinted at earlier. 
ExAMPLE 1.16 ([20], [15], [18]). Let $S^{3}=\left\{\left(r_{1} e^{i \theta_{1}}, r_{2} e^{i \theta_{2}}\right) \mid r_{1}^{2}+r_{2}^{2}=1\right\}$, and let $f$ : $\mathbf{R} P^{3} \rightarrow \mathbf{R} P^{3}$ be induced by the map $\tilde{f}: S^{3} \rightarrow S^{3}$ defined by $\tilde{f}\left(r_{1} e^{i \theta_{1}}, r_{2} e^{i \theta_{2}}\right)=$ $\left(r_{1} e^{3 i \theta_{1}}, r_{2} e^{3 i \theta_{2}}\right)$. Then $\pi_{1}\left(\mathbf{R} P^{3}\right)=\mathbf{Z}_{2}, f_{*}: \pi_{1}\left(\mathbf{R} P^{3}\right) \rightarrow \pi_{1}\left(\mathbf{R} P^{3}\right)$ is the identity, and $L\left(f^{m}\right)=1-9^{m} \neq 0$ for all $m$. From the sequence in $(1) \mathcal{R}\left(f_{*}^{m}\right) \cong \mathbf{Z}_{2}$ for each $m$, and for each $[\alpha]^{m} \in \mathcal{R}\left(f_{*}^{m}\right),\left\langle[\alpha]^{m}\right\rangle=\left\{[\alpha]^{m}\right\}$. That is since $f_{*}$ is the identity each orbit has length 1 . Now let $n=2^{r}$ for some positive integer $r$. Since for any $m \mid n$ the number $n / m$ must be even, it is not hard to see (compare 1.15) that $\iota_{m, n}$ is multiplication by an even integer, that is, it is the zero function on $\mathbf{Z}_{2}$. In particular the orbit $\left\langle[1]^{n}\right\rangle$ is irreducible and essential. From the definition $N P_{n}(f)=n=2^{r}$.

Note (looking ahead slightly) in this example that $N P_{n}(f)=n \cdot \#\left(I E C_{n}(f)\right.$ ) (see $1.19(\mathrm{v}))$, and by 1.19 , for $r>1$ that

$$
N \Phi_{n}(f) \geq \sum_{m \mid n} N P_{m}(f) \geq N P_{n}(f)=n>N\left(f^{n}\right) .
$$

We remark that in this example we needed to consider the algebra in order to determine that the essential class represented by $[\alpha]^{n}$ is irreducible. This is not detectable from the geometry alone.

1.4. Properties and estimates of $N \Phi_{n}(f)$ and $N P_{n}(f)$. In this subsection we give some properties of the periodic numbers. The ordinary Nielsen number $N(f)$ is a homotopy invariant, homotopy type invariant etc. These are the usual properties one expects of a Nielsen theory. Accordingly we have the following properties for the periodic Nielsen numbers.

THEOREM $1.17([20])$. The numbers $N P_{n}(f)$ and $N \Phi_{n}(f)$

(i) are homotopy invariants,

(ii) are homotopy type invariants for maps,

(iii) satisfy the commutative property of maps.

The next theorem is the expected lower bound property for our numbers.

THEOREM $1.18([20])$. $N P_{n}(f) \leq M P_{n}(f)$ and $N \Phi_{n}(f) \leq M \Phi_{n}(f)$.

Note that, in 1.1, $f$ has $N \Phi_{4}(f)=80=\#\left(\Phi\left(f^{4}\right)\right)$, and $N P_{4}(f)=72=\#\left(P_{4}(f)\right)$. Thus from theorem 1.18 we have that $N \Phi_{4}(f)=M \Phi_{4}(f)$ and $N P_{4}(f)=M P_{4}(f)$.

Next we have a number of estimates from various sources.

THEOREM 1.19. (i) ([20]) $N \Phi_{n}(f) \geq N \Phi_{m}(f)$ for all $m \mid n$,

(ii) ([18]) $N \Phi_{n}(f) \geq N\left(f^{m}\right)$ for all $m \mid n$,

(iii) ([20]) $N \Phi_{n}(f) \geq N \Phi_{m}\left(f^{n / m}\right)$ for all $m \mid n$,

(iv) ([20]) $N \Phi_{n}(f) \geq \sum_{m / n} N P_{m}(f)$,

(v) ([28]) \#(IEC $\left.C_{n}(f)\right) \leq N P_{n}(f) \leq n \cdot \#\left(I E C_{n}(f)\right)$.

For any given inequality in theorem 1.19 an example may be given where the inequality is strict, and another where the inequality is an equality (most such examples can be found in [15] and [18]). For (v) the left hand bound is attained in example 1.15, and the right hand one in 1.16. Necessary and sufficient conditions for any given inequality being an equality are illusive. Sufficient conditions can form the substance of computational theorems. 
2. Computational theorems and calculations. In this section we deal with the question of the computation of $N \Phi_{n}(f)$ and $N P_{n}(f)$. This, as might be expected, is even harder than the computational question for $N(f)$. As with ordinary Nielsen theory there are three fundamental tools, namely Jiang spaces, fibre space techniques, and the Fox calculus for surfaces. We document the progress in the use of these tools for the computation of $N \Phi_{n}(f)$ and $N P_{n}(f)$. In addition we also indicate something of how equivariant fixed point theory has contributed to periodic point theory.

2.1. Jiang spaces. In ordinary Nielsen theory the concept of being a Jiang space is a useful tool. We know for example that if $X$ is a Jiang space then for any $f: X \rightarrow X$

$$
N(f)= \begin{cases}0 & \text { if } L(f)=0, \\ \mathcal{R}(f) & \text { otherwise. }\end{cases}
$$

For periodic points the relationship is more subtle. The first result is due to Jiang. Let $k=\#$ Coker $\left(1-f_{*}\right)$ in homology (see sequence $(2)$ ).

Theorem 2.1 ([20]). Let $X$ be a Jiang space, and $f: X \rightarrow X$ a map.

(i) If $n$ has no prime factors other than those of $k$, and if $L\left(f^{n}\right) \neq 0$ then $N P_{n}(f) \geq$ $k \phi(n)$ where $\phi$ is the Euler function in number theory.

(ii) If $k>1$ and $L\left(f^{n}\right) \neq 0$ for all $n$, then $f$ has an infinite number of periodic points.

This theorem reflects Jiang's interest in such question as the existence of an infinite number of periodic points (see [21]). For this (ii) is clearly useful. However when we restrict our interest to fixed $n$ the lower bound given in (i) might not be too sharp!

EXAMPLE 2.2. In example 1.15 we see that $k=\#\left(\operatorname{Coker}\left(1-f_{*}\right)\right)=2$ and $\phi(4)=2$, so $k \phi(4)=4$. While, as we have seen, $N P_{4}(f)=72$.

Part of the cause of the problem with the inadequacy of this estimation of $N P_{n}(f)$ is to be found in the method of proof of 2.1 which used mod $K$ versions of periodic point numbers. Here $K$ is the kernel of the composite of the function $\pi_{1}(X) \rightarrow \mathcal{R}\left(f_{*}^{n}\right)$ with the function $\mathcal{R}\left(f_{*}^{n}\right) \rightarrow \operatorname{Coker}\left(1-f_{*}^{n}\right)$ induced by the Hurewicz homomorphism. This has the effect of coalescing a number of periodic points into a mod $K$ class (or orbit) and essentially counting the coalesced classes (orbits) as a single class (orbit).

2.2. Mainly for tori. In this subsection in order that we might give a smoother exposition, the results are, at times, out of chronological order.

Definition 2.3 ([12]). We say that $f$ is essentially reducible provided that for any essential class $[\alpha]^{n}$ of $f^{n}$, if $[\alpha]^{n}$ reduces to some class $[\beta]^{m}$, then $[\beta]^{m}$ is also essential. If for a given space $X$ any self map is essentially reducible, then we say that $X$ is essentially reducible.

The maps in examples 1.15 and 1.16 are essentially reducible while the one in example 1.8 is not. If a map of a Jiang space (as in example 1.8) is to be essentially reducible, we need that if $L\left(f^{n}\right) \neq 0$, then $L\left(f^{m}\right) \neq 0$ for any $m \mid n$, In particular by consideration of roots of unity we have

Proposition $2.4([12 ; 4,3])$. Tori are essentially reducible. 
Essential reducibility has a number of important consequences including

THEOREM 2.5 ([18; 4.2]). If $f$ is essentially reducible then

$$
N \Phi_{n}(f)=\sum_{m \mid n} N P_{m}(f)
$$

The point is that in the presence of essential reducibility the number $\sum_{m \mid n} N P_{m}(f)$ detects all periodic points detectable by current Nielsen theory. In other words, in terms of the definition, for essentially reducible maps, the union of the sets of irreducible essential orbits at each level $m \mid n$ is a set of $n$ representatives of $f$.

EXAmple 2.6 (see [20], [15]). For $f: \mathbf{R} P^{3} \rightarrow \mathbf{R} P^{3}$ and $n=2^{r}$ as in example 1.16 we have

$$
N \Phi_{n}(f)=\sum_{t=1}^{r} N P_{2^{t}}(f)=\sum_{t=1}^{r} 2^{t}=2^{r+1}=2 n .
$$

Essential reducibility also enables us to use either the $N \Phi_{n}(f)$ or the $N P_{m}(f)$ to determine the other, by Möbius inversion.

Corollary 2.7 ([18;4.6]). Let $f$ and $n$ be given, and let $\mathbf{p}(n)$ denote the set of prime divisors of $n$, and where $n: \tau=n \prod_{p \in \tau} p^{-1}$. If $f$ is essentially reducible then

$$
N P_{n}(f)=\sum_{\tau \subset \mathbf{p}(n)}(-1)^{\# \tau} N \Phi_{n: \tau}(f) .
$$

In order to motivate next the two main computational results from [15] and [18], we turn back to example 1.15. We noted there that $N \Phi_{n}(f)=N\left(f^{n}\right)$. We highlight some of the ingredients which make this equality work. We list a number of properties, (P1) to (P6), that were not in fact delineated like this until [12]. This labeling will also be useful later.

The reader can hardly fail to have noticed in example 1.15 (unlike 1.16) that we get the same number when we count $n$ times the number of irreducible essential orbits, as when we count irreducible essential classes. This is expressed in terms of length $\ell$ of an orbit, and depth $d$ of an orbit, as

(P1) For any $[\alpha]^{m}$, we have that $\ell\left([\alpha]^{m}\right)=d\left([\alpha]^{m}\right)$.

For the second property we have (unlike example 1.16)

(P2) The functions $\iota_{k, m}$ are injective for all $k|m| n$.

When all orbits are essential (P2) shows that orbits 'down below' may as well be counted at level $n$. For the third property we have

(P3) The maps $f$ are essentially reducible.

(P4) The map $f$ is reducible to the GCD, that is, whenever a class $[\alpha]^{m}$ of $f^{m}$ reduces to classes $[\beta]^{r}$ at level $r$ and to $[\delta]^{s}$ at level $s$, then there is a class $[\sigma]^{(r, s)}$ at level $(r, s)$ to which both $[\beta]^{r}$ and $[\delta]^{s}$ reduce.

A root of a periodic point class $[\alpha]^{n}$ is an irreducible periodic point class $[\beta]^{m}$ to which $[\alpha]^{n}$ reduces. Note that the next property holds for 1.1 , but not for 1.2 or 1.16 .

(P5) (Unique roots) Every class $[\alpha]^{m}$ of $f^{m}$ has a unique root $[\beta]^{r}$. 
Note that the injectivity of the $\iota$ does not insure unique roots since this only shows that if the images of two roots from the same level are equal then they are the same root. The next property holds for Jiang spaces.

(P6) For $f: X \rightarrow X$ we have either $N\left(f^{m}\right)=0$, or $N\left(f^{m}\right)=\mathcal{R}\left(f^{m}\right)$.

For spaces with abelian fundamental group (i.e. if $X$ is a Jiang space) (P4) and (P5) are automatic ([20; p. 66] and $[8 ; 4.9])$. In addition if $\pi_{1}$ is abelian, the first two properties (P1) and (P2) follow if Fixf $f_{*}^{n} \cong \operatorname{ker}\left(f_{*}^{n}-1\right) \cong 0$ (see sequence (2) or [15; 3.5]). This follows for tori if the matrix determined by $f$ on the first homology group does not have an $m t h$ root of unity for any $m \mid n$, i.e. if $L\left(f^{m}\right) \neq 0$. In addition for tori all the other necessary conditions are satisfied, so we have

Theorem $2.8([18 ; 4.12])$. Suppose that $X=T^{k}$ is a $k$-torus. If $L\left(f^{n}\right) \neq 0$ then

$$
N \Phi_{n}(f)=N\left(f^{n}\right) \text {. }
$$

ExAmple 2.9. Let $f: T^{24} \rightarrow T^{24}$ be a map of the 24, torus with characteristic equation $\lambda^{24}+1=0$ (it is not hard to find a matrix which will induce this map). Since $L\left(f^{24}\right) \neq 0$ we have from the theorem that $N \Phi_{24}(f)=N\left(f^{24}\right)=2^{24}$ (the last equality follows from $[15 ; 3.9])$.

Combining 2.4 and 2.7 with the last theorem we get the following special case of one of the main theorems of [15] proved there by combinatorial arguments. Note in contrast to corollary 2.7 that the $N P_{n}(f)$ are defined in terms of the ordinary Nielsen numbers $N\left(f^{n: \tau}\right)$ rather than the $N \Phi_{n: \tau}(f)$

Corollary 2.10 ([15;3.7]). Suppose that $X=T^{k}$ is a $k$-torus, and let $\mathbf{p}(n)$ denote the set of prime divisors of $n$. If $L\left(f^{n}\right) \neq 0$ then

$$
N P_{n}(f)=\sum_{\tau \subset \mathbf{p}(n)}(-1)^{\# \tau} N\left(f^{n: \tau}\right)
$$

where $n: \tau=n \prod_{p \in \tau} p^{-1}$.

EXAMPLE 2.11. For the map $f$ in example 2.9, $\mathbf{p}(n)=\{2,3\}$, so we have

$$
N P_{12}(f)=N\left(f^{12}\right)-N\left(f^{6}\right)-N\left(f^{4}\right)+N\left(f^{2}\right)=4020 .
$$

Here we use the formula $N\left(f^{m}\right)=2^{(m, n)}$ from [15;3.9] for calculating the ordinary Nielsen numbers.

We next show that the formula in corollary 2.10 is wrong if $L\left(f^{n}\right)=0$.

EXAMPLE 2.12. In example 2.9 we have $N P_{48}(f)=0$ since $L\left(f^{48}\right)=N\left(f^{48}\right)=0$. On the other hand the right hand side of the formula in corollary 2.10 is $0-N\left(f^{24}\right)-$ $N\left(f^{16}\right)+N\left(f^{12}\right)$ which is negative!

The same phenomena can also be seen from 1.2. For tori an algorithm for this situation was given in [18]. We need some notation. Define $M(f, n)$ by $M(f, n)=\left\{m|n| N\left(f^{m}\right) \neq 0\right.$, and if $m|k| n$ with $m \neq k$, then $\left.N\left(f^{k}\right)=0\right\}$. In example 2.9 since $N\left(f^{48}\right)=0$ and $N\left(f^{24}\right) \neq 0$, we have $M(f, 48)=\{24\}$. 
TheOREM $2.13([18 ; 4.7])$. Let $f: T^{k} \rightarrow T^{k}$ be a map of the $k$-torus. Then

$$
N \Phi_{n}(f)=\sum_{\emptyset \neq \mu \subseteq M(f, n)}(-1)^{\# \mu-1} N\left(f^{\xi(\mu)}\right),
$$

where $\xi(\mu)$ is the greatest common divisor of all elements of $\mu$.

EXAMPLE 2.14. In example 2.9, $N \Phi_{48}(f)=N\left(f^{24}\right)=2^{24}$, and in example 1.2, $N \Phi_{2}(f)=N(f)=2$.

Actually these results quoted above from [15] and [18] are a bit more general than we have stated them here. However we are not aware of any significant class of spaces for which the more general hypothesis in [15] and [18] are satisfied. Those hypotheses as they stand are certainly not valid for the class of spaces discussed in the next subsection.

2.3. Nil and solvmanifolds. We mentioned earlier that the first results about lower bounds for $M \Phi_{n}(f)$, and $M P_{n}(f)$ were suggested by Halpern in [4] for the Klein bottle. The Klein bottle is an example of a solvmanifold (a homogeneous space of a solvable Lie group). It seems worth while quoting his result here. We need first to define some maps on the Klein bottle.

ExAmple 2.15 (The Klein bottle). Let $K^{2}$ denote the Klein bottle. We will think of $K^{2}$ as a quotient space of $\mathbf{R}^{2}$, its universal covering space. For the fundamental domain $[0,1] \times[0,1] \subseteq \mathbf{R}^{2}$ this results in the familiar identifications of $(0, s)$ with $(1,1-s)$ and $(t, 0)$ with $(t, 1)$ (for more details see [14]). Given any pair of integers $(r, q)$ for which $r$ is odd, or $r$ is even and $q=0$, the correspondence $(s, t) \rightarrow(r s, q t) \bmod \mathbf{Z}^{2}$ induces a well defined map $f$ of $K^{2}$. We shall call this map $f$ the standard $(r, q)$ map on $K^{2}$.

Halpern starts his preprint [4] by saying that the object of his paper is to determine the minimum number of periodic points (i.e. $M \Phi_{n}(f)$ ) for all maps of $K^{2}$. He very quickly goes on to state that his result is the following (when applied to the set of maps defined above)

TheOREM 2.16 ([4]). For $n \geq 1$, the Nielsen numbers $N\left(f^{n}\right)$ for $f$ the standard $(r, q)$ map on $K^{2}$ are given by

$$
N\left(f^{n}\right)= \begin{cases}\left|q^{n}\left(r^{n}-1\right)\right| & \text { if } q \neq 0 \\ \left|\left(r^{n}-1\right)\right| & \text { if } q=0 .\end{cases}
$$

Thus Halpern implicitly equated $M \Phi_{n}(f)$ with $N\left(f^{n}\right)$. We saw in 1.2 that this can be quite inadequate. Part of our aim in this subsection is to show what is true in equating $M \Phi_{n}(f)$ with $N\left(f^{n}\right)$ for nil and solvmanifolds, and what is not.

2.3A. Equivariant fixed point theory and periodic points. The main tools concerning nilmanifolds (homogeneous spaces of nilpotent Lie groups) and solvmanifolds (homogeneous spaces of solvable Lie groups) are fibre techniques (see the next subsection for more details). However the first results about periodic points on nilmanifolds were given by Peter Wong ([28]) using equivariant Nielsen theory, and in particular his inequality $\#\left(I E C_{n}(f)\right) \leq N P_{n}(f) \leq n \cdot \#\left(I E C_{n}(f)\right)$ (see 1.19(iv)).

We sketch the connection between equivariant fixed point theory that enabled Wong to give his results, and to discover the above inequality (see [27]). We use an example. 
Let $f: X \rightarrow X$ be given; we consider periodic points of period 3. The first step is to construct the space $X_{3}=X \times X \times X$ and the cyclic $\mathbf{Z}_{3}$ action which under the operation by the first non-zero element takes a triple $\left(x_{1}, x_{2}, x_{3}\right)$ to $\left(x_{3}, x_{1}, x_{2}\right)$.

Consider next the $\mathbf{Z}_{3}$ map $g_{f}: X_{3} \rightarrow X_{3}$ given by $g_{f}\left(x_{1}, x_{2}, x_{3}\right)=\left(f\left(x_{3}\right), f\left(x_{1}\right), f\left(x_{2}\right)\right)$. For $\left(x_{1}, x_{2}, x_{3}\right)$ to be a fixed point of $g_{f}$ we must have

$$
\left(x_{1}, x_{2}, x_{3}\right)=\left(f\left(x_{3}\right), f\left(x_{1}\right), f\left(x_{2}\right)\right) .
$$

That is, $f\left(x_{3}\right)=x_{1}, f\left(x_{1}\right)=x_{2}$ and $f\left(x_{2}\right)=x_{3}$, or

$$
f^{3}\left(x_{1}\right)=f^{2}\left(x_{2}\right)=f\left(x_{3}\right)=x_{1} .
$$

In other words the set of periodic points of period 3 can be identified with the $\mathbf{Z}_{3}$ equivariant fixed point set of $g_{f}$ on $X_{3}$. More generally $n$-periodic point theory can be considered as $\mathbf{Z}_{n}$ equivariant fixed point theory on $X_{n}$, the $n$-fold product. Thus by applying the machinery of equivariant fixed point theory to the spaces $X_{n}$, we obtain information about periodic point theory.

As an example of what Wong proved we give the following very specific theorem for nilmanifolds.

THEOREM 2.17 ([28]). Let $f: N \rightarrow N$ be a map of a compact nilmanifold, $p$ a prime number, $n=p^{r}$, and $m=p^{r-1}$. Suppose that $\iota_{m, n}$ is injective. If $L\left(f^{n}\right) \cdot L\left(f^{m}\right) \neq 0$, then

$$
\left|L\left(f^{n}\right)\right|-\left|L\left(f^{m}\right)\right| \leq N P_{n}(f) \leq n \cdot\left(\left|L\left(f^{n}\right)\right|-\left|L\left(f^{m}\right)\right|\right)
$$

With the extra requirement that $f$ satisfies (P1), Wong shows that the equality can be settled as the left hand value. As we see below this is proved (and generalized in several ways) without hypotheses in [12].

2.3B. Fibre techniques on nil and solvmanifolds. The results of this (sub)-subsection use fibre techniques, as do the computation of the ordinary Nielsen numbers of nil and solvmanifolds. We report on two papers [12] and [13]. The first of these aims to show for which maps of nilmanifolds and solvmanifolds one can generalize theorems 2.8, 2.10 and 2.13. The second aims to develop algorithms for the cases when these theorems cannot be applied.

The properties of nil and solvmanifolds that make them amenable to the use of fibre techniques is the toral decomposition that these spaces have. We will let $K=k e r i_{*}$ : $\pi_{1}(F) \rightarrow \pi_{1}(E)$, and $N_{K}\left(f_{p(y)}\right)$ be the $\bmod K$ Nielsen number (e.g. [14]).

Definition 2.18 ([14]). A fibration $F \hookrightarrow E \stackrel{p}{\rightarrow} B$ is said to satisfy the naïve addition conditions provided that for each fibre-preserving map $f$ of $p$, and for all $y$ in an essential class of $f$ we have that $F i x \bar{f}_{*}^{p(y)}=p_{*}\left(\operatorname{Fix} f_{*}^{y}\right)$ and $N_{K}\left(f_{p(y)}\right)=N\left(f_{p(y)}\right)$.

THEOREM 2.19 ([2]). Let $N$ be a nilmanifold which is not a torus. Then there is a sequence of orientable fibrations $T_{k} \hookrightarrow N \rightarrow N_{k}, T_{k-1} \hookrightarrow N_{k} \rightarrow N_{k-1}, \ldots, T_{3} \hookrightarrow N_{2} \rightarrow$ $N_{1}, T_{2} \hookrightarrow N_{1} \rightarrow T_{1}$, where $N_{1}, N_{2}, \ldots, N_{k-1}, N_{k}, N_{k+1}=N$ are nilmanifolds and $T_{1}$, $T_{2}, \ldots, T_{k}$ are tori. For solvmanifolds $S$, there is one more fibration $N_{k+1} \rightarrow S \rightarrow T_{k+1}$ in the tower. All these fibrations satisfy the naïve addition conditions. Furthermore any self map of $N$ (or $S$ ) is homotopic to a map which is fibre preserving with respect to this decomposition. 
See 2.26 for an example of a nilmanifold that is not a torus. We call the tower of fibrations in 2.19 the toral decomposition of $N$ or $S$. Note that for solvmanifolds the last fibration may not be orientable (e.g. 2.15).

Let $f: E \rightarrow E$ be a fibre preserving self map of a fibration $F \stackrel{j}{\hookrightarrow} E \stackrel{p}{\rightarrow} B$, with induced map $\bar{f}$ on the base $B$. Consider the following diagrams, where the base point $x$ of $E$ is an element of $\Phi\left(f^{m}\right), b=p(x)$, and $\left(f^{m}\right)_{b}$ is the restriction of $f^{m}$ to the fibre $F=p^{-1}(b)$.

\begin{tabular}{|c|c|c|c|c|}
\hline $\mathcal{E}\left(\left(f^{m}\right)_{b}\right)$ & $\subseteq \mathcal{R}\left(\left(f^{m}\right)_{b *}\right)$ & $\mathcal{R}\left(\left(f^{m}\right)_{b *}\right)$ & $\stackrel{\iota}{\longrightarrow}$ & $\mathcal{R}\left(\left(f^{n}\right)_{b *}\right)$ \\
\hline$j_{*} \downarrow$ & $j_{*} \downarrow$ & $j_{*} \downarrow$ & & $j_{*} \downarrow$ \\
\hline $\mathcal{E}\left(f^{m}\right)$ & $\subseteq \mathcal{R}\left(f_{*}^{m}\right)$ & $\mathcal{R}\left(f_{*}^{m}\right)$ & $\stackrel{\iota}{\longrightarrow}$ & $\mathcal{R}\left(f_{*}^{n}\right)$ \\
\hline$p_{*} \downarrow$ & $p_{*} \downarrow$ & $p_{*} \downarrow$ & & $p_{*} \downarrow$ \\
\hline $\mathcal{E}\left(\bar{f}^{m}\right)$ & $\subseteq \mathcal{R}\left(\bar{f}_{*}^{m}\right)$ & $\mathcal{R}\left(\bar{f}_{*}^{m}\right)$ & $\stackrel{\iota}{\rightarrow}$ & $\mathcal{R}\left(\bar{f}_{*}^{n}\right)$ \\
\hline
\end{tabular}

Proposition 2.20. The diagrams above are commutative, and the vertical sequences are exact in the sense that ker $p_{*}=\operatorname{Im} j_{*}$. In particular if $[\alpha]^{n} \in \mathcal{R}\left(f_{*}^{n}\right)$ is such that $p_{*}\left([\alpha]^{n}\right)$ is the basepoint of $\mathcal{R}\left(\bar{f}_{*}^{n}\right)$, and $\left[\alpha_{b}\right]^{n}$ in the fibre is such that $j_{*}\left(\left[\alpha_{b}\right]^{n}\right)=[\alpha]^{n}$ then from the left hand diagram $[\alpha]^{n}$ is essential if and only if the components $p_{*}\left([\alpha]^{n}\right)$ and $\left[\alpha_{b}\right]^{n}$ of $[\alpha]^{n}$ are.

The commutativity follows by naturality considerations, the exactness from the longer sequences from [7].

Proposition 2.21. Let $f$ be a map of a nilmanifold or solvmanifold then (P3) holds, i.e. $f$ is essentially reducible; moreover properties (P1), (P2), (P4) and (P5) hold on essential classes.

Perhaps the easiest property to understand is the essential version of (P1), that the $\iota$ are injective on essential classes. By 2.19 we may assume all maps are fibre preserving and we proceed by induction on the toral decomposition. The induction hypothesis is that the $\iota$ are injective on essential classes in fibre and base (note the tower of fibrations in 2.19 starts with tori in both base and fibre). The inductive step for the essential version of (P1) is proved by 'five lemma' type arguments. Note that the $j_{*}$ in 2.19 are not in general injective. However since these fibrations satisfy the naïve addition conditions we have from [14] that $j_{*}: \mathcal{R}\left(\left(f^{m}\right)_{* b}\right) \rightarrow \mathcal{R}\left(f_{*}^{n}\right)$ is injective on essential classes. Observe that there is also an extra difficultly in the proofs because we are dealing with exact sequences of sets not groups!

If the reader will allow us to gloss over the existence of suitable base points (which is far from trivial), we can also give the flavour of the proof of (P3). Here the induction hypothesis is that fibre and base are essentially reducible. Take an essential class $[\alpha]^{n} \in \mathcal{R}\left(f_{*}^{n}\right)$, and suppose it reduces to $[\beta]^{m}$. By $2.20, p_{*}\left([\alpha]^{n}\right)$ is essential, and by the commutativity of the bottom part of the diagram there, $p_{*}\left([\alpha]^{n}\right)$ is reducible to $p_{*}\left([\beta]^{m}\right)$. By the inductive hypothesis $p_{*}\left([\beta]^{m}\right)$ is essential and thus contains a point $b \in \Phi\left(\bar{f}^{m}\right)$. This will act as a new basepoint for $B$, and ensure that the restriction $\left(f^{m}\right)_{b}$ of $f^{m}$ to $p^{-1}(b)$ is a self map of the fibre. By change of basepoint we can assume that $p_{*}\left([\beta]^{m}\right)$ is the neutral element of $\left.\mathcal{R}\left(\left(\bar{f}^{m}\right)\right)\right)$, so $[\beta]^{m}$ has a fibre component $\left[\beta_{b}\right]^{m}$ in $\left.\mathcal{R}\left(\left(f^{m}\right)_{b}\right)\right)$. By 
commutativity of the diagrams in $2.20, \iota\left(\left[\beta_{b}\right]^{m}\right)$ is a fibre component of $[\alpha]^{n}$. Since $[\alpha]^{n}$ is essential, its components are essential by 2.20 . By the inductive hypothesis the fibre and base are essentially reducible and so the components of $[\beta]^{m}$ are essential. Again by $2.20,[\beta]^{m}$ itself is essential as required.

Definition 2.22. If $f: X \rightarrow X$ has the property that $N\left(f^{m}\right)=0$, or $N\left(f^{m}\right)=$ $\mathcal{R}\left(f^{m}\right)$ then we call $f^{m}$ weakly Jiang. If for a given $X$ every self map is weakly Jiang we say that $X$ itself is weakly Jiang.

The class of $\mathcal{N} \mathcal{R}$ solvmanifolds is a large class of solvmanifolds that includes all exponential solvmanifolds where the exponential map is onto.

Proposition 2.23. Any map of a nilmanifold or $\mathcal{N} \mathcal{R}$ solvmanifold is weakly Jiang. For a map $f: S \rightarrow S$ of an arbitrary solvmanifold, $f^{m}$ is weakly Jiang if and only if for each $b$ in an essential class of the induced map $\bar{f}^{m}$, we have $N\left(\left(f^{m}\right)_{b}\right)=0$, or $N\left(\left(f^{m}\right)_{b}\right) \neq 0$.

The last part of the proposition gives the clue to the proof that again uses the commutative diagrams of the type of diagram found in 2.20 . The discerning reader will note that is it the fibre uniformity of nil and $\mathcal{N} \mathcal{R}$ solvmanifolds that allow for them to be weakly Jiang. As we see in the next example most (but not all) maps of the Klein bottle are weakly Jiang (but not fibre uniform).

EXAMPLE 2.24. Let $K^{2}$ be the Klein bottle, and $f$ the standard $(r, q)$ map defined in 2.15. Now $K^{2}$ fibres over $S^{1}$ as the fibration $S^{1} \hookrightarrow K^{2} \stackrel{p}{\rightarrow} S^{1}$ where $p$ is projection on the second factor. Moreover with respect to this fibration the map $f$ is fibre preserving.

For the iterates of $f$ we have that $f^{n}$ is the standard $\left(r^{n}, q^{n}\right)$ map with induced map $\bar{f}^{n}$ of degree $r^{n}$ on the base. We denote the fixed point set of the induced map $\bar{f}^{n}$ on the base by $\Phi\left(\bar{f}^{n}\right)=\left\{x_{j}: j=0,1, \ldots,\left|r^{n}-1\right|-1\right\}$, where $x_{0}=\{0,1\}$ in $S^{1}=I /[0 \sim 1]$, and for $j \neq 0$ the points $x_{j}=j /\left|1-r^{n}\right|$ are equally spaced on the circle. The Nielsen numbers on the various fibres are given by $N\left(f_{x_{j}}^{n}\right)=\left|1-(-1)^{j} q^{n}\right|$ (see [14]). Note that if $q \neq \pm 1$ then these numbers are all non-zero for all $j$, and for all $n$, but if $q= \pm 1$ then some of these numbers are zero and some not. Thus by 2.23 the map $f$ is weakly Jiang for all $n$ if and only if $q \neq \pm 1$.

In conjunction with the condition that $N\left(f^{m}\right)=\mathcal{R}\left(f^{m}\right)$ we see that properties (P1)(P5) will hold on all classes. Thus we have the following generalization of 2.8 and 2.10.

TheOREM 2.25 ([12; 1.2]). Suppose that $f: X \rightarrow X$ is an arbitrary map of a compact nilmanifold or $\mathcal{N} \mathcal{R}$ solvmanifold, or suppose $f$ is an arbitrary map of a compact solvmanifold and that $f^{n}$ is weakly Jiang. If $N\left(f^{n}\right) \neq 0$ then

$$
N \Phi_{n}(f)=N\left(f^{n}\right) \text { and } N P_{n}(f)=\sum_{\tau \subset \mathbf{p}(n)}(-1)^{\# \tau} N\left(f^{n: \tau}\right)
$$

with the same notation as 2.10 .

Theorem 2.13 can also be generalized (see [12]). Since for nil and $\mathcal{N} \mathcal{R}$ solvmanifolds $N\left(f^{n}\right)=\left|L\left(f^{n}\right)\right|$ theorem 2.25 settles Wong's inequality 2.17 as

$$
N P_{n}(f)=\left|L\left(f^{n}\right)\right|-\left|L\left(f^{m}\right)\right| \text {. }
$$


We give two examples of 2.25, the first is dubbed Baby Nil in [12] since it is the smallest nilmanifold that is not a torus.

EXAMPLE 2.26. Let $G$ be the topological group that is represented by matrices over the reals of the form (left hand matrix)

$$
\left(\begin{array}{ccc}
1 & x & y \\
0 & 1 & z \\
0 & 0 & 1
\end{array}\right) \quad \phi\left(\begin{array}{ccc}
1 & x & y \\
0 & 1 & z \\
0 & 0 & 1
\end{array}\right)=\left(\begin{array}{ccc}
1 & 2 x & 6 y \\
0 & 1 & 3 z \\
0 & 0 & 1
\end{array}\right) .
$$

The binary operation is matrix multiplication. Let $\Gamma$ be the discrete subgroup consisting of those elements of $G$ with integer entries. Then $M=G / \Gamma$ is a nilmanifold. There is one fibration in the toral decomposition for $M$, namely $S^{1} \rightarrow M \stackrel{p}{\rightarrow} S^{1} \times S^{1}$, where $p$ is induced by the projection on the " $x$ " and " $z$ " factors.

Let $\phi: G \rightarrow G$ be defined as displayed above. Note that $\phi$ is a homomorphism and that $\phi$ takes $\Gamma$ to itself. Thus $\phi$ induces a map $f: M \rightarrow M$, and it is naturally fibre preserving. Since $p$ is orientable and satisfies the naïve addition conditions we have the product formula $N\left(f^{n}\right)=N\left(f_{0}^{n}\right) N\left(\bar{f}^{n}\right)$ for the ordinary Nielsen number $N\left(f^{n}\right)$. Thus by 2.25

$$
N \Phi_{n}(f)=N\left(f^{n}\right)=\left|\left(1-6^{n}\right)\left(1-2^{n}\right)\left(1-3^{n}\right)\right| .
$$

As a special case, for $n=12$, we also have from theorem 2.25 that

$$
\begin{aligned}
N P_{12}(f) & =N\left(f^{12}\right)-N\left(f^{6}\right)-N\left(f^{4}\right)+N\left(f^{2}\right) \\
& =4,737,215,588,698,939,920 .
\end{aligned}
$$

ExAmple 2.27 (The Klein bottle). Let $K^{2}$ be the Klein bottle, and $f$ the standard map of type $(r, q)$ (see 2.15). For $q \neq \pm 1$ we have from 2.25 and 2.24

$$
N \Phi_{n}(f)=N\left(f^{n}\right)= \begin{cases}\left|q^{n}\left(r^{n}-1\right)\right| & \text { if } q \neq 0 \\ \left|\left(r^{n}-1\right)\right| & \text { if } q=0 .\end{cases}
$$

Thus modulo the periodic Wecken question on the Klein bottle, Halpern's suggestion that the formula above gives $M \Phi_{n}(f)$ is correct except for the case $q \neq \pm 1$ (see 2.24).

The next result from [13] also uses fibre techniques, and is useful for maps on solvmanifolds when $f^{n}$ is not weakly Jiang.

Theorem 2.28 ([11], [13]). Let $E$ be a solvmanifold, and $f: E \rightarrow B$ be a fibre preserving map of the minimal Mostow fibration for E. Suppose furthermore that $\#\left(\Phi\left(\bar{f}^{n}\right)\right)$ $=N\left(\bar{f}^{n}\right)$. Then

$$
N P_{n}(f)=\sum_{b \in \Phi\left(\bar{f}^{n}\right)} N P_{n / \operatorname{per}(b)}\left(f_{b}^{p e r(b)}\right) .
$$

Our example of 2.28 (repeated below from [13]) shows that for the Klein bottle with $q= \pm 1$ it is $N P_{m}(f)$ that is equal to $N\left(f^{m}\right)$, rather than $N \Phi_{n}(f)$ as might have been expected (see [13] and its announcement [11] and Halpern's claim in [4] and at the beginning of this subsection).

EXAMPLE 2.29. Let $K^{2}$ denote the Klein bottle, let $r$ be odd, we consider the standard $(r,-1)$ map $f$ of $K^{2}$. The induced map $\bar{f}^{n}$ of the $n$th iterate on the base of the 
standard fibration $S^{1} \hookrightarrow K^{2} \stackrel{p}{\rightarrow} S^{1}$ is the standard map of degree $r^{n}$. Thus as in 2.27, $\Phi\left(\bar{f}^{n}\right)=\left\{x_{j}|j=0,1, \ldots,| r^{n}-1 \mid-1\right\}$. From 2.15 the maps on the fibre alternate with degree +1 on even indices, and -1 on odd indices. We calculate $N P_{n}(f)$ for $n=2^{k}$ for all positive integers $k$.

On $S^{1}$ maps of degree \pm 1 have the following $N P$ numbers:

$$
N P_{n}\left(1_{S^{1}}\right)=N\left(1_{S^{1}}\right)=0, \text { and } N P_{n}\left(-1_{S^{1}}\right)= \begin{cases}1 & \text { if } n=1 \\ 0 & \text { otherwise. }\end{cases}
$$

Now all reducible points of $\bar{f}^{n}$ have even indices, and those that do not have the $N P$ numbers equal to 0 , so

$$
N P_{n}(f)=\sum_{j \text { odd }} N P_{1}\left(\left(f^{n}\right)_{x_{j}}\right)=\sum_{x_{j} \in \Phi\left(\bar{f}^{n}\right)} N P_{1}\left(\left(f^{n}\right)_{x_{j}}\right)=\sum_{x_{j} \in \Phi\left(\bar{f}^{n}\right)} N\left(\left(f^{n}\right)_{x_{j}}\right)=N\left(f^{n}\right) .
$$

The second step is because over the even indices $N P_{1}\left(\left(f^{n}\right)_{x_{j}}\right)$ is zero, the third by definition, and the fourth by the naïve addition formula [14, 24]. We note that in this case $N\left(f^{n}\right)=N\left(\bar{f}^{n}\right)=\left|1-r^{n}\right|$.

Thus for $n=2^{k}$ we get the perhaps surprising result that $N P_{n}(f)$ (rather than $\left.N \Phi_{n}(f)\right)$ is equal to $N\left(f^{n}\right)$.

This example shows that Halpern's suggestion that $N\left(f^{n}\right)$ gives the formula for $M \Phi_{n}(f)$ is incorrect in the case $q= \pm 1$.

2.4. The Fadell-Husseini method and the Fox calculus. The final method of calculation of the Nielsen number that we mention is the so called Fadell-Husseini method for surfaces. It employs the Fox calculus together with a number of other techniques, and uses the Reidemeister trace $R(f, \tilde{f})$ of a self map $f$ of a closed, non-simply connected surface $X$. For the periodic point numbers we report on recent work of Hart and Keppelmann in [6], mainly on the double torus or handcuff space $T^{2} \# T^{2}$. This work uses and extends (to the periodic point numbers) an improvement on these methods given by Davey, Hart and Trapp ([2]).

The computational results in ordinary Nielsen theory in this area consist mainly of examples, and refinements of the method. In other words computational theorems are sparse, and individual calculations are made using a variety of techniques (for example abelianization). Accordingly one would not expect deep computational theorems in the extension of these methods to periodic point theory. The explorations mentioned in the title of the work on which we are reporting ([6]) examine the properties (P1) through (P6), show that in the general case we cannot expect the type of theorem found for nil and solvmanifolds, but exhibit an example of a homeomorphism where, though not all of the essential versions of (P1) through (P6) hold, nevertheless $N \Phi_{n}(f)=N\left(f^{n}\right)$ and $N \Phi_{n}(f)=\sum_{m \mid n} N P_{m}(f)$ for all $n$.

We introduce the necessary machinery only for $T^{2} \# T^{2}$. Let $\pi=\pi_{1}\left(T^{2} \# T^{2}\right)=$ $\langle a, b, c, d: R\rangle$, with $R=a b a^{-1} b^{-1} c d c^{-1} d^{-1}$. Because $T^{2} \# T^{2}$ is a $K(\pi, 1)$ every endomorphism $\phi: \pi \rightarrow \pi$ is induced by a self map $f$ on $T^{2} \# T^{2}$. Thus we may consider endomorphisms of $\pi$, rather than continuous maps.

The Reidemeister trace of $f^{n}$ is described as follows (the $\tilde{f}^{n}$ in the notation denotes a chosen lift of $f^{n}$, which as remarked in section 1.2 is for us equivalent to choosing a 
base point in $X)$.

$$
R\left(f^{n}, \tilde{f}^{n}\right)=\tau_{n}\left(1-\frac{\partial \phi^{n}(a)}{\partial a}-\frac{\partial \phi^{n}(b)}{\partial b}-\frac{\partial \phi^{n}(c)}{\partial c}-\frac{\partial \phi^{n}(d)}{\partial d}+A_{n}\right),
$$

where $\tau_{n}: \mathbf{Z}[\pi] \rightarrow \mathbf{Z}\left(\mathcal{R}\left(f_{*}^{n}\right)\right)$ is defined by extending linearly the function that for each $\alpha \in \pi$ is given by $\tau_{n}(\alpha):=[\alpha]^{n}$; and where the derivatives are those of the Fox calculus which provides a partial derivative for each generator of $\pi$ as

$$
\frac{\partial x_{i}}{\partial x_{j}}=\delta_{i, j}, \quad \frac{\partial 1}{\partial x_{j}}=0
$$

and for $u, v \in F$,

$$
\frac{\partial u v}{\partial x_{j}}=\frac{\partial u}{\partial x_{j}}+u \frac{\partial v}{\partial x_{j}},
$$

and where $A_{n}$ is defined by the following algorithm. Write $\phi(R)$ in the form $\prod_{i=1}^{r} y_{i} R^{\lambda_{i}} y_{i}^{-1}$ for $r \in \mathbf{Z}^{+}, \lambda_{i} \in \mathbf{Z}$, and $y_{i} \in \pi$ for each $i$. Then $A_{n}=\sum_{i}\left(\lambda_{i} y_{i}\right) \in \mathbf{Z}[\pi]$. (We ask the experts' indulgence for the oversimplification we have made by ignoring the fact that the Fox calculus is defined on the free group $F=\langle a, b, c, d\rangle$, rather than $\pi$ ).

The Reidemeister trace of $f^{n}$ incorporates information about both the Nielsen classes and their indices, into an algebraic object. When $R\left(f^{n}, \tilde{f}^{n}\right)$ has been reduced so that each Reidemeister class appears at most once, then the coefficient of each Reidemeister class is its index. Thus the essential classes are exactly the classes with non-zero coefficients in the reduced form, and $N\left(f^{n}\right)$ is the number of such terms.

The main difficulty in the calculation of $N\left(f^{n}\right)$ by this method is in the reduction of the Reidemeister trace. A similar difficulty appears in calculating the periodic numbers. A number of tools are available none of which is algorithmic. One such tool is abelianization This employs the function $\theta: \mathcal{R}\left(f_{*}^{n}\right) \rightarrow \operatorname{Coker}\left(1-f_{*}^{n}\right)$ induced by the Hurewicz homomorphism (see sequence (2) in section 1). From sequence (2) we see that $\operatorname{Coker}\left(1-f_{*}^{n}\right) \cong \mathbf{Z}^{4} / \operatorname{Im}\left(f_{*}^{n}-1\right)$. The subgroup $\operatorname{Im}\left(f_{*}^{n}-1\right)$ can be described in terms of a matrix. The image under $\theta$ is the coset of a 4-tuple whose coordinates are the sums of the exponents of the generators. For example $\theta\left(b^{-3} a^{2} b^{2} a^{-1}\right)$ is the coset of $(1,-1,0,0)$. In example 2.31 below we see by abelianization, that $\theta\left([1]^{2}\right)$ and $\theta\left(\left[b^{3}\right]^{2}\right)$ determine the same coset of $\operatorname{Im}\left(f_{*}^{n}-1\right.$ ) (use the matrix of $f_{*}^{n}-1$ ). This shows that $[1]^{2}$ and $\left[b^{3}\right]^{2}$ may determine the same Reidemeister class. A word search reveals they do (see 2.31). Abelianization shows that $[1]^{2},[b]^{2}$ and $\left[b^{2}\right]^{2}$ are distinct. For the periodic point numbers we consider the diagram below (note that there is a technical difficulty which for the sake of a smooth exposition we will ignore, and that is that in [6] the boosting functions are backwards from ours).

$$
\begin{array}{lll}
\mathcal{R}\left(f_{*}^{m}\right) & \stackrel{\iota_{m, n}}{\longrightarrow} & \mathcal{R}\left(f_{*}^{n}\right) \\
\theta \downarrow & & \theta \downarrow \\
\mathbf{Z}^{4} / \operatorname{Im}\left(f_{*}^{m}-1\right) & \stackrel{\bar{\iota}_{m, n}}{\longrightarrow} & \mathbf{Z}^{4} / \operatorname{Im}\left(f_{*}^{n}-1\right) .
\end{array}
$$

There is also a similar diagram involving $\theta$, and the maps induced by $f$ on $\mathcal{R}\left(f_{*}^{n}\right)$ and $\mathbf{Z}^{4} / \operatorname{Im}\left(f_{*}^{n}-1\right)$. The next proposition is an easy consequence of the commutativity of the 
diagram above. The concepts of abelian length and abelian depth are just the obvious ones on $\mathbf{Z}^{4} / \operatorname{Im}\left(f_{*}^{n}-1\right)$.

Proposition 2.30 ([6]). The diagrams mentioned above are commutative, and moreover (abelian) depth $\leq$ (ordinary) depth, and (abelian) length $\leq$ (ordinary) length.

We give first an example where $f$ is neither essentially reducible, nor weakly Jiang, nevertheless at least for some $n, N \Phi_{n}(f)=N\left(f^{n}\right)$.

EXAMPLE $2.31([6])$. Let $\phi$ be the endomorphism given by $\phi(a)=a b^{-1} c, \phi(b)=b^{-2}$, $\phi(c)=b^{-2}$ and $\phi(d)=a b^{-1} c$. Then $A_{1}=0$ and

$$
R(f, \tilde{f})=\tau_{1}\left(1-1+b^{-1}+b^{-2}\right)=0[1]^{1}+\left[b^{-1}\right]^{1}+\left[b^{-2}\right]^{1} .
$$

The classes $\left[b^{-1}\right]^{1}$ and $\left[b^{-2}\right]^{1}$ are distinct and different from $[1]^{1}$ by abelianization. At level $2, A_{2}=0$, and because $\left[b^{3}\right]^{2}=\left[\phi^{2}(b) b^{-1}\right]^{2}=[1]^{2}$,

$$
R\left(f^{2}, \tilde{f}^{2}\right)=-[1]^{2}-[b]^{2}-\left[b^{2}\right]^{2}-\left[b^{3}\right]^{2}=-2[1]^{2}-[b]^{2}-\left[b^{2}\right]^{2} .
$$

The last expression is in reduced form by abelianization. Note that $\left[\iota_{1,2}(1)\right]^{2}=[1]^{2}$, $\left[\iota_{1,2}\left(b^{-1}\right)\right]^{2}=[b]^{2}$, and $\left[\iota_{1,2}\left(b^{-2}\right)\right]^{2}=\left[b^{2}\right]^{2}$. So the class $[1]^{2}$ is essential and reduces to the inessential class $[1]^{1}$. Thus $T^{2} \# T^{2}$ does not satisfy property (P3), essential reducibility. In particular $3=N \Phi_{2}(f) \neq N P_{1}(f)+N P_{2}(f)=2+0$ (see 2.5).

Since $N(f)=2$, and $[1]^{1}$ is inessential, $f$ is not weakly Jiang (P6), nevertheless at least for $n=2$, we do get that $N \Phi_{2}(f)=3=N\left(f^{2}\right)$.

A similar but more involved example is given ([6; example 3]) where properties of the essential versions of (P2) and (P5) are not satisfied, i.e. $T^{2} \# T^{2}$ does not have unique roots and the $\iota$ are not always injective on essential classes. In this case a hands on argument shows that $N \Phi_{2}(f) \neq N\left(f^{2}\right)$. Finally the authors give the following example of an automorphism of $\pi$ which we may assume is induced by a homeomorphism:

EXAMPLE $2.32([6])$. Let $\phi$ be the automorphism given by $\phi(a)=b^{-1} a^{-1}, \phi(b)=a b^{2}$, $\phi(c)=d$, and $\phi(d)=c$. It turns out for any homeomorphism inducing $\phi$ that the Nielsen numbers are very closely related to the Lucas numbers, and that essential classes 'boost' to essential classes. Among other properties, we note that each essential class has exactly one essential root, but may have inessential roots. Interestingly although $f^{n}$ is never weakly Jiang, nevertheless there are enough properties around to allow the authors to deduce that $N \Phi_{n}(f)=N\left(f^{n}\right)$. This is a highly technical, case specific proof. It depends very heavily on being able to write the Reidemeister classes in terms of products of $\phi^{j}(a)$ for different powers $j$. In this example essential reducibility also holds, so $N \Phi_{n}(f)=\sum_{m \mid n} N P_{n}(f)$.

Example 2.32 is interesting for a number of reasons. Firstly because it is proved without requiring, as is the case for nil and solvmanifolds, that all the "essential versions" of axioms (P1) through (P6) in section 2.2 are satisfied. In fact not all these axioms hold since as pointed out already, for no $n$ is the map $f^{n}$ weakly Jiang. In other words conditions (P1) to (P6) are sufficient, but neither they nor the essential versions of them given in [12] are necessary. Secondly the example shows that homeomorphisms, as in the fixed point case, may be a species of their own. Such thoughts led the authors to a more technical version the following conjecture: 
Conjecture 2.33. Let $f: M \rightarrow M$ be a homeomorphism on a surface $M$ of nonpositive Euler characteristic. Then $N \Phi_{n}(f)=\sum_{m \mid n} N P_{n}(f) s$, and when $N\left(f^{n}\right) \neq 0$, then $N \Phi_{n}(f)=N\left(f^{n}\right)$.

3. Restricted classes. In this section we report on two theories of restricted classes of periodic points. The first concerns a relative theory which parallels relative fixed point theory which originated with Schirmer [25]. The second is fibred periodic point theory which parallels the fibred fixed point theory given for ordinary Nielsen theory introduced in [8], and generalized to the non-fibre uniform situation in [14].

3.1. Relative periodic point numbers. The definition of the ordinary relative Nielsen number $N(f ; X, A)$ of a self map $f:(X, A) \rightarrow(X, A)$ combines the fixed point classes of $f$ and its restriction. It is

$$
N(f ; X, A)=N\left(f_{A}\right)+N(f)-N\left(f ; f_{A}\right),
$$

where $f_{A}$ is the restriction of $f: X \rightarrow X$ to the subspace $A$, and where $N\left(f ; f_{A}\right)$ is the number of essential fixed point classes of $f$ that contain an essential fixed point class of $f_{A}$. This number $N(f ; X, A)$ was introduced to give a more accurate lower bound for the number of fixed points on the restricted class of maps (and homotopies of such) that are invariant on the subspace $A$. Such a number was needed because the ordinary Nielsen number may be a poor lower bound for the restricted class of maps of pairs of spaces. Not surprisingly the same phenomena occur with periodic points.

In relative theory we are often considering manifolds with nonconnected boundary as the subspace. We give an example of a pair of spaces (with disconnected subspace) where we might want to know the minimum number of periodic points. This example is dubbed the Banana example in [17] and [16] from which the results of this subsection are taken.

EXAMPLE 3.1. Let $X$ be the solid torus $X=S^{1} \times D^{2}$. A self map $f$ of $X$ is defined by describing first its restriction to the 'core' $S^{1} \times\{0\}$ of $X$. This restriction, which will have the same homotopy type as a map to the whole space, is the standard map of degree 3 (see 1.1). The points $e^{j \pi i / 4} \times\{0\}$ for $j=0,1, \ldots, 7$ are fixed points of the restriction of $f^{2}$ to the core, and each point is in its own Nielsen class. For $j=0,4$ the classes are reducible. All the rest are irreducible, and all classes are essential.

Let $A=A_{0} \cup A_{1} \cup A_{2} \cup \ldots \cup A_{7}$ be the subspace of $X=S^{1} \times D^{2}$ in which $A_{j}$ is the boundary circles of the disks $\left\{e^{j \pi i / 4}\right\} \times D^{2}$, with $j=0,1, \ldots, 7$. Integers $d_{j}$ are selected as follows: $d_{0}=1 ; d_{1}=1, d_{3}=5 ; d_{2}=2, d_{6}=2 ; d_{4}=2 ; d_{5}=7, d_{7}=1$. We define the restriction $f_{j}: A_{j} \rightarrow A_{3 j}$ (3j is taken $\left.\bmod 8\right)$ of $f$ as follows. The map $f_{j}$ is the standard map of degree $d_{j}$ on $S^{1}$ for $j=0,1, \ldots, 7$. In other words $f_{A}$ maps each circle of $A$ to another circle of $A$ in a fashion compatible with the map $f$. Next we extend $f$ to the whole space to obtain a map $f:(X, A) \rightarrow(X, A)$ by defining $f$ on each of the eight pieces $\left\{e^{i \theta} \times D^{2}: j \pi / 4 \leq \theta \leq(j+1) \pi / 4\right\}$ of $X$. Each such piece is shaped like a piece of a banana, and the map $f$ maps each piece into the union of three pieces so that each piece is stretched according to the map $f$ on the core, and 'twisted' according to the map $f_{A}$ until a continuous map $f:(X, A) \rightarrow(X, A)$ (determined up to homotopy) is obtained. 
For a given map $f:(X, A) \rightarrow(X, A)$ we define $\widehat{A}$ to be the union of the components of $A$ that are mapped back into themselves by $f_{A}^{k}$ for some integer $k$. For each $A_{i} \in \widehat{A}$ we define the cycle length $c(i)$ of $A_{i}$ to be the least integer $k$ for which $f_{A}^{k}\left(A_{i}\right) \subseteq A_{i}$. This divides $\widehat{A}$ into equivalence classes called cycles. In example 3.1 the $f_{A^{-c y c l e s}}$ of $A$ are $\left[A_{0}\right]=\left\{A_{0}\right\},\left[A_{1}\right]=\left\{A_{1}, A_{3}\right\},\left[A_{2}\right]=\left\{A_{2}, A_{6}\right\},\left[A_{4}\right]=\left\{A_{4}\right\}$ and $\left[A_{5}\right]=\left\{A_{5}, A_{7}\right\}$.

For the relative Nielsen number $N(f ; X, A)$ the extension of Nielsen theory to a nonconnected space $A$ presents no problem. For periodic points the situation is not quite so simple. In example 3.1 if $i \neq 0$ or 4 unless the period of $f^{n}$ is even, then $f_{A}^{n}\left(A_{i}\right) \mid A_{i}$ is not even a self map of $A_{i}$, We use $f_{j}^{k}$ to denote the restriction of $f_{A}^{k}$ to $A_{j}$. In 3.1

$$
\Phi\left(f_{A}^{2 n}\right)=\Phi\left(\left(f_{0}\right)^{2 n}\right) \cup \Phi\left(\left(f_{1}^{2}\right)^{n}\right) \cup \ldots \cup \Phi\left(\left(f_{4}\right)^{2 n}\right) \cup \ldots \cup \Phi\left(\left(f_{7}^{2}\right)^{n}\right) .
$$

The point being that for $j \neq 0,4, f_{j}$ is not a self map of $A_{j}$, but $f_{j}^{2}$ is. It should be clear that

$$
N \Phi_{2 n}\left(f_{A}\right)=N \Phi_{2 n}\left(f_{0}\right)+N \Phi_{n}\left(\left(f_{1}^{2}\right)\right)+\ldots+N \Phi_{2 n}\left(f_{4}\right)+\ldots+N \Phi_{n}\left(\left(f_{7}^{2}\right)\right)
$$

is a lower bound for the number or periodic points on $A$ of all periods less than or equal to $n$. Thus a reasonable definition for $N P_{n}\left(f_{A}\right)$ and $N \Phi_{n}\left(f_{A}\right)$ on a non-path-connected space $A$ would be as follows:

DEFINITION 3.2.

$$
N P_{n}\left(f_{A}\right)=\sum_{j} N P_{n / c(j)}\left(f_{k}^{c(j)}\right) \text { and } N \Phi_{n}(f A)=\sum_{j} N \Phi_{n / c(j)}\left(f_{k}^{c(j)}\right)
$$

where the sums are taken over those $j$ for which $A_{j} \in \widehat{A}$, and $c(j) \mid n$.

The numbers $N \Phi_{n}\left(f_{A}\right)$ and $N P_{n}\left(f_{A}\right)$ satisfy all the same properties $N P_{n}(f)$ and $N \Phi_{n}(f)$ satisfy on connected spaces (see section 1.4).

EXAMPLE 3.3. In 3.1 by the commutative law $N\left(f_{1}^{2}\right)=N\left(f_{3}^{2}\right), N\left(f_{2}^{2}\right)=N\left(f_{6}^{2}\right)$ and $N\left(f_{5}^{2}\right)=N\left(f_{7}^{2}\right)$. Thus we see for example using 2.8 that

$$
\begin{aligned}
N \Phi_{2}\left(f_{A}\right) & =N\left(\left(f_{0}\right)^{2}\right)+2 N\left(f_{1}^{2}\right)+2 N\left(f_{2}^{2}\right)+N\left(\left(f_{4}\right)^{2}\right)+2 N\left(f_{5}^{2}\right) \\
& =0+2|1-5|+2|1-4|+\left|1-2^{2}\right|+2|1-7|=29,
\end{aligned}
$$

and similarly $N \Phi_{1}\left(f_{A}\right)=1, N \Phi_{3}\left(f_{A}\right)=7$, and $N \Phi_{6}\left(f_{A}\right)=1121$.

In 3.1 by the nonconnected version of 2.10 we also have for example from above that $N P_{2}(f)=29-1=28$, and

$$
\begin{aligned}
N P_{6}\left(f_{A}\right) & =N \Phi_{6}\left(f_{A}\right)-N \Phi_{3}\left(f_{A}\right)-N \Phi_{2}\left(f_{A}\right)+N \Phi_{1}\left(f_{A}\right) \\
& =1121-7-29+1=1086 .
\end{aligned}
$$

For a self map $f:(X, A) \rightarrow(X, A)$, let $N P_{n}\left(f, f_{A}\right)$ be the number of irreducible essential orbits of $f$ that contain an essential orbit of $f_{A}$.

Definition 3.4. Let $f:(X, A) \rightarrow(X, A)$ be a map of a pair of compact ANR's. The relative Nielsen type number of period $n$ is

$$
N P_{n}(f ; X, A)=N P_{n}\left(f_{A}\right)+N P_{n}(f)-N P_{n}\left(f, f_{A}\right) .
$$


ExAMPLE 3.5. Consider 3.1. For $n=2$ we get

$$
\begin{aligned}
N P_{2}(f ; X, A) & =N P_{2}\left(f_{A}\right)+N P_{2}(f)-N P_{2}\left(f, f_{A}\right) \\
& =28+6-6=28 .
\end{aligned}
$$

To see that $N P_{2}\left(f, f_{A}\right)=6$, we use the definition and notice that for $j=1,2,3,5,6$ and 7 the map $f_{j}^{2}$ has at least one fixed point which is Nielsen equivalent to the 2-periodic point of $f: X \rightarrow X$ which corresponds to $e^{j \pi i / 4}$.

For $n=6$ there are no common essential irreducible orbits of $f$ and $f_{A}$, and so $N P_{6}\left(f, f_{A}\right)=0$. Thus (also using 2.10)

$$
\begin{aligned}
N P_{6}(f ; X, A) & =N P_{6}\left(f_{A}\right)+N P_{6}(f)-N P_{6}\left(f, f_{A}\right) \\
& =1086+\left|1-3^{6}\right|-\left|1-3^{3}\right|-\left|1-3^{2}\right|+|1-3|-0=1782 .
\end{aligned}
$$

By analogy with the ordinary Nielsen type number $N \Phi_{n}(f)$, the definition of the relative Nielsen type number $N \Phi_{n}(f ; X, A)$ requires of us that we generalize the idea of a set of $n$-representatives. Such a set interconnects the orbits of $f$ and those of $f_{A}$ at various levels. It is not written quite so easily as $N P_{n}(f ; X, A)$. We do not give the full definition of $N \Phi_{n}(f ; X, A)$ but observe that it has many of the properties of the ordinary periodic Nielsen numbers, for example

Theorem 3.6. $N \Phi_{n}(f ; X, A) \geq \sum_{m \mid n} N P_{m}(f ; X, A)$, and if $f$ and $f_{j}^{c(j)}$ are essentially reducible for all $j$, then equality holds.

Let $M P_{n}(f ; X, A)$ and $M \Phi_{n}(f ; X, A)$ be the obvious minimum numbers. Then

$$
N P_{n}(f ; X, A) \leq M P_{n}(f ; X, A) \text {, and } N \Phi_{n}(f ; X, A) \leq M \Phi_{n}(f ; X, A) .
$$

Also $N P_{n}(f ; X, A) \geq N P_{n}\left(f_{A}\right)$ and $N \Phi_{n}(f ; X, A) \geq N \Phi_{n}\left(f_{A}\right)$, and

$$
N P_{n}(f ; X, A) \geq N P_{n}(f) \text { and } N \Phi_{n}(f ; X, A) \geq N \Phi_{n}(f) \text { etc. }
$$

Our final result gives conditions under which the relative Nielsen number for the $n$th iterate of a map of pairs $f:(X, A) \rightarrow(X, A)$ equals the relative Nielsen number of $f^{n}$. These conditions are clearly satisfied in example 3.1 .

Theorem 3.7. If $f:(X, A) \rightarrow(X, A)$ is a self map, then

$$
N \Phi_{n}(f ; X, A) \geq N\left(f^{n} ; X, A\right) .
$$

Furthermore, if $f$ and $f_{j}^{c(j)}$ satisfies properties (P1) through (P6), then equality holds.

In [17] an example is given where the inequality in 3.7 is strict. This example called the 'projective banana', essentially mirrors 3.1 except that it has a subspace $A$ that consists of eight copies of $\mathbf{R} P^{3}$ (cf. example 1.16).

There are a great many extensions and refinements of relative Nielsen theory (see the survey article [26]). Potentially this is also true of relative periodic point theory. One such refinement in the fixed point case was introduced by Zhao [31], and concerns the location of fixed points. This can be illustrated by the relative map $f$ of the pair $\left(D^{2}, S^{1}\right)$, in which $f$ is a small irrational rotation. There is a single fixed point of $f$ which happens to lie in $D^{2}-S^{1}$. This is detected by the relative Nielsen number of $f$ which is 1 . However this fixed point may be moved to $S^{1}$ by a relative homotopy. That is, the fixed point may be located in either $A$ or the complement $X-A$. This example shows that the relative 
Nielsen number of a self map $f$ of a pair $(X, A)$ gives no information about location of fixed points, and in particular it gives no information on the minimum number of fixed points on the complement. Zhao's work in [31] refines relative theory by considering weakly common fixed point classes. Taking our gross oversimplification of the existence of fixed points of a map even further, let us further assume (again for expositional reasons) that there is a fixed point $x_{0}$ of $f$ in $A$ (this oversimplification will be untenable when $A$ has more than one component). Then there is a function $i_{*}: \mathcal{R}\left(f_{A *}\right) \rightarrow \mathcal{R}\left(f_{*}\right)$ induced by the inclusion $i: A \rightarrow X$. A class is said to be weakly common if it is the image of $i_{*}$. Zhao's number $N(f ; X-A)$ is the number of essential classes of $f$ on $X$ which are not weakly common. In the proper technical version of this, the single fixed point class of $f$ and $f_{S^{1}}$ in the illustration is essential weakly common. This demonstrates that weakly common fixed point classes may have empty intersection. The number $N(f ; X-A)$ is shown in [31] to be a lower bound for the number of fixed points on $X-A$ (and is 0 in the example). The bound is sharp under the usual dimensional restrictions, when paths with endpoints in $X-A$ can be pulled off $A$ by relative homotopies (the bypassing condition). We illustrate briefly a generalization of all this to periodic point theory. The reference for the generalization is [19] which also discusses briefly the scope and difficulty of other generalizations and extensions of relative fixed point theory. The example is the following:

ExAmple 3.8. Let $X=S^{1} \times S^{1}=\left\{\left(e^{\theta i}, e^{\phi i}\right) \mid 0 \leq \theta \leq 2 \pi, 0 \leq \phi \leq 2 \pi\right\}$, and let $A=\Delta S^{1}=\left\{\left(e^{\theta i}, e^{\phi i}\right) \mid \theta=\phi\right\}$ be the diagonal of $X$. We define a relative map $f:(X, A) \rightarrow$ $(X, A)$ by $f\left(e^{\theta i}, e^{\phi i}\right)=\left(e^{2 \phi i}, e^{2 \theta i}\right)$. For $n$ odd the periodic points for this map on $X$ are given by

$$
\Phi\left(f^{n}\right)=\left\{\left(e^{i \theta}, e^{i \phi}\right) \mid \theta=\frac{2 j \pi}{2^{2 n}-1}, \phi=\frac{2^{n+1} j \pi}{2^{2 n}-1}, j=0,1, \ldots, 2^{2 n}-2\right\}
$$

and for $n$ even

$$
\Phi\left(f^{n}\right)=\left\{\left(e^{i \theta}, e^{i \phi}\right) \mid \theta=\frac{2 j_{1} \pi}{2^{n}-1}, \phi=\frac{2 j_{2} \pi}{2^{n}-1}, j_{1}, j_{2}=0,1, \ldots, 2^{n}-2\right\} .
$$

The name of the game, for this example for a given $n$, is to give a sharp lower bound for the numbers $\left.M \Phi\left(f^{n} ; X-A\right)=\min \#\left((X-A) \cap\left\{\Phi\left(g^{n}\right)\right) \mid g \sim_{A} f\right\}\right)$ (the minimum numbers of periodic points of all periods dividing $n$ of maps $g$ that are homotopic to $f$ as a map of pairs, and that lie in $X-A)$, and for $M P_{n}(f ; X-A)=\min \#\left((X-A) \cap\left\{P_{n}(g)\right) \mid g \sim_{A}\right.$ $f\}$ (the minimum numbers of periodic points of period exactly $n$ of maps $g$ that are homotopic to $f$ as a map of pairs, and that lie in $X-A)$. Note that homotopies must be both relative homotopies (that is homotopies of pairs), and homotopies of $f$ rather than $f^{n}$. The challenge in this, and similar examples, is not to determine which points might or might not be combined under relative homotopies of $f$, for this follows from existing Nielsen theory. The challenge is to determine which period point classes (or more precisely orbits) may be moved from $X-A$ to $A$ under such relative homotopies. The numbers defined in [19] are denoted by $N \Phi_{n}(f ; X-A)$ and $N P_{n}(f ; X-A)$. We will not 
attempt to define them here. However in [19] it is shown for 3.8 that

$$
N \Phi_{n}(f ; X-A)=M \Phi_{n}(f ; X-A)= \begin{cases}2^{2 n}-3 \cdot 2^{n}+2 & \text { if } n \text { is even } \\ 2^{2 n}-2^{n} & \text { otherwise. }\end{cases}
$$

Furthermore $M P_{m}(f ; X-A)=N P_{m}(f ; X-A)$, and these latter numbers can be obtained by (now familiar) Möbius type inversion formulae. Some insight for the even case can be gleaned for the facts that $X$ and $A$ are Jiang spaces; that for examples like this, $N \Phi_{n}(f ; X-A)=N\left(f^{n} ; X-A\right)$; that $N\left(f^{n}\right)=\mathcal{R}\left(f^{n}\right)=\#\left(\mathbf{Z}_{2^{n}-1} \times \mathbf{Z}_{2^{n}-1}\right)$; that $i_{*}: \mathcal{R}\left(f_{S^{1} *}^{n}\right) \rightarrow \mathcal{R}\left(f_{*}^{n}\right)$ is injective; and finally that $\mathcal{R}\left(f_{S^{1}}^{n}\right)=\#\left(\mathbf{Z}_{2^{n}-1}\right)=2^{n}-1$. For insight into why $N \Phi_{n}(f)=M \Phi_{n}(f)$ see theorem 4.1.

3.2. Fibred periodic point numbers. We briefly review fibred fixed point theory. Let $f: E \rightarrow E$ be a fibre preserving map of a fibration $p: E \rightarrow B$ with induced map $\bar{f}$ on the base. If $b \in B$ is a fixed point of $\bar{f}$ then $f$ restricts to a give a self map $f_{b}$ of the fibre $F_{b}=p^{-1}(b)$. The fibred Nielsen number of $f$ (introduced in [8] and generalized to the non-fibre uniform situation in [14]) is defined to be

$$
N_{\mathcal{F}}(f, p)=\sum_{b \in \chi} N\left(f_{b}\right),
$$

where $\chi$ consists of a representative of each essential class of $\bar{f}$ (or later of $\bar{f}^{n}$ ). The number $N_{\mathcal{F}}(f, p)$ has the property that

$$
N(f) \leq N_{\mathcal{F}}(f, p) \leq M_{\mathcal{F}}(f, p),
$$

where $M_{\mathcal{F}}(f, p)$ is the minimum number of fixed points within the fibre homotopy class of $f$. The point is that the first inequality may be strict (see 3.14), and under mild conditions equality holds in the second.

Let $F_{\chi}=p^{-1}(\chi) \subset E$, and note that it is a disjoint union of fibres, i.e.

$$
F_{\chi}=\bigcup_{b \in \chi} F_{b} .
$$

Thus $f$ can be considered as a self map of the pair $\left(E, F_{\chi}\right)$, i.e. $f:\left(E, F_{\chi}\right) \rightarrow\left(E, F_{\chi}\right)$. The next theorem relates the fibred Nielsen number and the relative Nielsen numbers.

TheOREm 3.9. $N_{\mathcal{F}}(f, p)=N\left(f ; E, F_{\chi}\right)=\Sigma_{b \in \chi} N\left(f_{b}\right)$, and $N_{\mathcal{F}}(f, p)=N(f)$ if and only if $f$ satisfies the naïve addition conditions (see 2.18).

In words the second part shows that under the naïve addition conditions the relative and the fibred and the ordinary Nielsen numbers of a fibre preserving map coincide, and these numbers can be calculated as a simple addition formula. The aim of this subsection is to do something similar for the periodic numbers. This work was reported in [9]. As an application an alternative proof of a theorem from [13] is given. For ease of exposition for the rest of this subsection, we will make the following oversimplification:

$$
\#(\chi)=\#\left(\Phi\left(\bar{f}^{n}\right)\right)=N\left(\bar{f}^{n}\right) .
$$

Motivated by 3.9 we make the following definition.

Definition 3.10. Let $B$ be essentially reducible. Then

$$
N P_{n}^{\mathcal{F}}(f, p)=N P_{n}\left(f ; E, F_{\chi}\right) .
$$


Let $M P_{n}^{\mathcal{F}}(f, p)$ be the minimum number of periodic points within the fibre homotopy class of $f$. The point of the definition, as with all Nielsen theories, is the lower bound property.

THEOREM 3.11. Let $f: E \rightarrow E$ be a fibre preserving map with $B$ essentially reducible. Then $M P_{n}^{\mathcal{F}}(f, p) \geq N P_{n}^{\mathcal{F}}(f, p)$.

The equation $N\left(f ; E, F_{\chi}\right)=\sum_{b \in \chi} N\left(f_{b}\right)$ for the fixed point case in 3.9 depends on the fact that every fixed point class of $f$ contains a fixed point class of the fibre (i.e. $N(f)=N\left(f, f_{\chi}\right)$, where $\left.f_{\chi}=f \mid p^{-1}(\chi)\right)$. There is a similar identification in the periodic point case which allows the next theorem in which $N P_{n}\left(f_{\chi}\right)$ is the periodic point number on the non-connected space $F_{\chi}$ (see definition 3.2). We need to understand what the $f$ cycles are here, but this is easy because $c\left(F_{b}\right)$, the length of the cycle of $F_{b}$, is simply $\operatorname{per}(b)$, the period of $b$.

THEOREM 3.12. If $f$ is a fibre preserving map with $B$ essentially reducible then $N P_{n}(f)=N P_{n}\left(f, f_{\chi}\right)$, so

$$
N P_{n}^{\mathcal{F}}(f, p)=N P_{n}\left(f_{\chi}\right)=\sum_{b \in \chi} N P_{n / p e r(b)}\left(f_{j}^{p e r(b)}\right) .
$$

The reader will recognize the formula from 2.28 in 3.12. By 3.2 and by analogy with 3.9 we expect more periodic points from our fibred periodic point number. The first part of the next result is a simple consequence of properties of the relative periodic point numbers (see section 3.1).

THEOREM 3.13. If $f: E \rightarrow B$ is a fibre preserving map with $B$ essentially reducible then

$$
N P_{n}^{\mathcal{F}}(f, p) \geq N P_{n}(f)
$$

and if $f^{m}$ satisfies the naïve addition conditions for appropriate $m$ then

$$
N P_{n}(f)=N P_{n}^{\mathcal{F}}(f, p)=\sum_{b \in \chi} N P_{n / p e r(b)}\left(f_{j}^{\text {per(b) }}\right)
$$

Thus under the naïve addition conditions we can calculate $N P_{n}(f)$ by the above addition formula. Not all fibre preserving maps satisfy the naïve addition conditions of course. The Hopf fibration gives a simple example where the inequality in 3.13 is strict.

ExAmple 3.14. Let $S^{1} \hookrightarrow S^{3} \stackrel{p}{\rightarrow} S^{2}$ be the Hopf fibration, and let $\bar{f}=d: S^{2} \rightarrow S^{2}$ be a map of degree $d$ where $d \neq \pm 1$, and $d \neq 2$. There is no obstruction to lifting $\bar{f}$ to a fibre preserving map $f: S^{3} \rightarrow S^{3}$ over $\bar{f}$. Since $N(\bar{f})=N\left(\bar{f}^{n}\right)=1, \bar{f}^{n}$ has only one point which must be a fixed point (by our oversimplification). We denote this point by $b(=\chi)$. Then $f$ induces, by restriction, a map $f_{b}: S^{1} \rightarrow S^{1}$ also of degree $d$. Clearly $\operatorname{per}(b)=1$, and by 3.13 and 2.1 (compare 1.15)

$$
N P_{n}^{\mathcal{F}}(f, p)=N P_{n}\left(f_{b}\right) \geq|1-d| \phi(n)>N P_{n}(f),
$$

where $\phi$ is the Euler function in number theory. The inequality holds since $\pi_{1}\left(S^{3}\right)=0$, so $N P_{1}(f)=N(f)=1$, and for $n>1, N P_{n}(f)=0$.

We see next and finally that theorem 2.28 is a corollary of 3.13 . The proof indicated here (which of course depends on 3.13) is more intuitive than the proof given in [13] 
(which came first). In fact the motivation for the work on fibre techniques in [12] and [13] came from an earlier version of 3.13 while it was still a conjecture (cf. [10] where a inadequate prototype for $N P_{n}^{\mathcal{F}}(f, p)$ was given).

Corollary 3.15 ([11], [13]). Let $E$ be a solvmanifold, and $f: E \rightarrow B$ be a fibre preserving map of the minimal Mostow fibration for $E$, and suppose that $\#(\chi)=\#\left(\Phi\left(\bar{f}^{n}\right)\right)=$ $N\left(\bar{f}^{n}\right)$. Then $N P_{n}(f)=\sum_{b \in \chi} N P_{n / \operatorname{per}(b)}\left(f_{j}^{\text {per }(b)}\right)$.

4. Periodic Wecken properties. For the ordinary Nielsen number we have that $N(f) \leq M(f)$, and the (ordinary) Wecken question asks under what conditions is there a map $g$ homotopic to $f$ with $\#(\Phi(g))=N(f)$, i.e. when is $N(f)=M(f)$ ? For the periodic numbers we have seen (1.18) that

$$
N \Phi_{n}(f) \leq M \Phi_{n}(f), \quad \text { and } \quad N P_{n}(f) \leq M P_{n}(f) .
$$

The periodic Wecken question then asks when these inequalities are actual equalities. In Jiang's book ([20; p. 71]) we have the following statement (as a theorem):

If $X$ is a compact connected differentiable manifold of dimension $\geq 5$, then $N \Phi_{n}(f)=$ $M \Phi_{n}(f)$, and $N P_{n}(f)=M P_{n}(f)$.

This result, which is attributed to Halpern, was put there on the strength of a private conversation that took place between Jiang and Halpern. No proof is given. This is a little worrying because Halpern had the wrong definition (the first naïve suggestion for $N P_{n}(f)$ - see section 1.1).

Wong in [28] has given a sketch proof of Halpern's 'theorem'. The basic idea is that there are Wecken theorems for equivariant fixed point theory and using the spaces $X_{n}$ (see section 2.3A) periodic point theory is a special case of the equivariant Wecken theorems.

Wong's sketch seems to need more details. Given the Wecken theorems for equivariant fixed point theory it is clear that for a self map $f$, of $X$, there is an equivariant map $k$ for which there is an equivariant homotopy $g_{f} \cong k$ (see section $2.3 \mathrm{~A}$ for the notation). However the details of why $k$ should be of the form $g_{\ell}$ for some $\ell$ are missing from the sketch, as is any indication of why, given the existence of such an $\ell$, the given homotopy should be induced by a homotopy $f \cong \ell$.

We do however have the following theorem due to Chengye You [29, 30].

Theorem 4.1 (You [29]). Let $f: T^{k} \rightarrow T^{k}$ be a map of the $k$-torus. Then $N \Phi_{n}(f)=$ $M \Phi_{n}(f)$ and $N P_{n}(f)=M P_{n}(f)$.

Apart from the above there is no significant progress on these questions. In particular no relative or fibred versions of the periodic Wecken question are forthcoming. In the fibred case an easy proof of ordinary (fixed point) Wecken properties of nil and solvmanifolds was given in [14]. This is based on the fact that for fixed points fibred and ordinary Wecken properties coincide under the naïve addition conditions. However generalization to the periodic Wecken situation is not straightforward and to date no progress has been made. 
5. Concluding comments. We conclude this survey by showing the relevance of the consideration of orbits (on which the definitions of $N \Phi_{n}(f)$ and $N P_{n}(f)$ are based) to the study of periodic points with all periods taken together. The following theorem is due to Halpern.

THEOREM 5.1 ([3]). If $f: T^{k} \rightarrow T^{k}$ is a map of the $k$-torus, then every map homotopic to $f$ has an infinite number of periodic points if and only if the sequence of Nielsen numbers $\left\{N\left(f^{n}\right)\right\}$ is unbounded.

In seeking to generalize such a theorem to other spaces, the existence of a close connection of $N \Phi_{n}(f)$ and $N P_{m}(f)$ with the various $N\left(f^{m}\right)$ plays a significant role (compare the results of [22] with those of section 2.3). In general however the boundedness of $\left\{N\left(f^{n}\right)\right\}$ implies neither the boundedness of $N \Phi_{n}(f)$ or of $N P_{n}(f)$, nor that $f$ is homotopic to a map with finitely many periodic points.

ExAmple 5.2. Let $f: \mathbf{R} P^{3} \rightarrow \mathbf{R} P^{3}$ be the map given in example 1.16. Then the sequence $\left\{N\left(f^{n}\right)\right\}$ is bounded, but the numbers $\left\{N P_{n}(f)\right\}$ are unbounded. So any map which is homotopic to $f$ must have an infinite number of periodic points.

Thus any study of all periods taken together must take into account the $N \Phi_{n}(f)$ and the $N P_{n}(f)$ numbers. In particular the study must deal at times with orbits rather than just with periodic point classes (see [21]).

\section{References}

[1] R. F. Brown, The Lefschetz Fixed Point Theorem, Scott, Foresman, and Company, 1970.

[2] O. DAvey, E. L. HART, and K. Trapp, Computation of Nielsen numbers for maps of closed surfaces, Trans. Amer. Math. Soc. 348 (1996), 3245-3266.

[3] B. Halpern, Periodic points on tori, Pacific J. Math. 83 (1979), 117-133.

[4] B. Halpern, Periodic points on the Klein bottle, never published.

[5] B. HALPERN, Nielsen type numbers for periodic points, never published.

[6] E. L. HART and E. C. KePPELmann, Explorations in Nielsen periodic point theory for the double torus, Topology Appl. 95 (1999), 1-30.

[7] P. R. HEAth, Product formulae for Nielsen numbers of fibre maps, Pacific J. Math. 117 (1985), 267-289.

[8] P. R. HEATH, A Nielsen type number for fibre-preserving maps, Topology Appl. 53 (1993), $19-35$.

[9] P. R. HeAth, Relating relative, fibred and absolute Nielsen numbers of periodic points, Talk given at the AMS meeting in Baton Rouge, Louisiana, April 19-21, 1996, special session on "Fixed point theory and dynamical systems"

[10] P. R. HEATH, Nielsen type numbers for fibre preserving maps, coincidences of fibre preserving maps, and for periodic points of fibre preserving maps, C. R. Math. 14 (1992), $25-30$.

[11] P. R. Heath and E. Keppelmann, Fibre techniques in Nielsen periodic point theory on nil and solvmanifolds, C. R. Math. 16 (1994), 229-234.

[12] P. R. Heath and E. Keppelmann, Fibre techniques in Nielsen periodic point theory on nil and solvmanifolds I, Topology Appl. 76 (1997), 217-247. 
[13] P. R. Heath and E. Keppelmann, Fibre techniques in Nielsen periodic point theory on nil and solvmanifolds II, to appear in Topology Appl.

[14] P. R. Heath, E. Keppelmann, and P. Wong, Addition formulae for Nielsen numbers and for Nielsen type numbers of fibre preserving maps, Topology Appl. 67 (1995), 133-157.

[15] P. R. Heath, R. Piccinini, and C. You, Nielsen type numbers for periodic points I, in: B. Jiang (ed.), Topological Fixed Point Theory and Applications, Lecture Notes in Math. 1411, Springer, Berlin, 1989.

[16] P. R. HeAth, H. SchiRmer, and C. You, Nielsen type numbers for periodic points on nonconnected spaces, Topology Appl. 63 (1995), 97-116.

[17] P. R. Heath, H. Schirmer, and C. You, Nielsen type numbers for periodic points on pairs of spaces, Topology Appl. 63 (1995), 117-138.

[18] P. R. HeAth and C. You, Nielsen type numbers for periodic points II, Topology Appl. 43 (1992), 219-236.

[19] P. R. Heath and X. Zhao, Periodic points on the complement, to appear in Topology Appl.

[20] B. JiAng, Lectures on Nielsen Fixed Point Theory, Contemp. Math. 14, Amer. Math. Soc., Providence, 1983.

[21] B. Jiang, Applications of the Nielsen theory to dynamics, these proceedings.

[22] E. Keppelmann, Periodic points on nilmanifolds and solvmanifolds, Pacific J. Math. 164 (1985), 105-128.

[23] E. Keppelmann and C. MCCond, The Anosov theorem for exponential solvmanifolds, Pacific J. Math. 170 (1995), 143-159.

[24] C. MCCORD, Nielsen numbers and Lefschetz numbers on solvmanifolds, Pacific J. Math. 147 (1991), 153-164.

[25] H. Schirmer, A relative Nielsen number, Pacific J. Math. 122 (1986), 459-473.

[26] H. Schirmer, A survey of relative fixed point theory, in: Nielsen Theory and Dynamical Systems (Mt. Holyoke, 1992), Contemp. Math. 152, 1993, 291-3309.

[27] P. Wong, Equivariant Nielsen numbers, Pacific J. Math. 159 (1993), 153-175.

[28] P. Wong, Fixed points on pairs of nilmanifolds, Topology Appl. 62 (1995), 173-179.

[29] C. You, A note on periodic points on tori, preprint.

[30] C. You, The least number of periodic points on tori, Adv. in Math. 24 (1995), 155-160.

[31] X. Zнао, A relative Nielsen number for the complement, in: Topological Fixed Point Theory and Applications (Tianjin, 1998), Lecture Notes in Math. 1411, Springer, 1989, $257-265$. 